\title{
Equilibrium between radiation and matter for classical relativistic multiperiodic systems. II. Study of radiative equilibrium with Rayleigh-Jeans radiation
}

\author{
R. Blanco, L. Pesquera, and E. Santos \\ Departamento de Física Teórica, Universidad de Santander, Santander, Spain
}

(Received 11 April 1983)

\begin{abstract}
We continue the study of the problem of equilibrium between radiation and classical relativistic systems begun previously [Phys. Rev. D $\underline{27}, 1254$ (1983)]. We consider the emission and absorption of energy by a relativistic pointlike particle immersed in a Rayleigh-Jeans radiation field. The particle is acted upon by a force which, if alone, would produce a multiply periodic motion. It is shown that radiative balance at each frequency holds. A discussion is given of the results reported in both papers.
\end{abstract}

\section{INTRODUCTION}

This is the second paper of a series dealing with the study of the equilibrium between radiation and classical relativistic material systems. In the first paper ${ }^{1}$ we showed that, if a relativistic multiperiodic system is immersed in a random radiation having a Rayleigh-Jeans spectrum at a given temperature, then the equilibrium distribution of the system is the Maxwell-Boltzmann distribution. In this paper we shall show that, under these conditions, the radiation field is also in equilibrium at each frequency. That is, the power emitted and the power absorbed by the system exactly cancel at each frequency on the average.

As is well known, the problem of the equilibrium between radiation and matter (i.e., the derivation of the blackbody spectrum) was actively studied at the beginning of the century. In the last few years there has been renewed interest in the subject. ${ }^{2}$ All previous studies were made in the nonrelativistic approximation. In this context, Boyer ${ }^{3}$ has shown that if we impose energy balance at each frequency and for each state of the mechanical system, we are led necessarily to the Rayleigh-Jeans (RJ) spectrum for the radiation and the Maxwell-Boltzmann (MB) distribution for the material system. The same conclusions are obtained ${ }^{4}$ if we impose the less stringent and more physical condition that the energy balance is satisfied at each frequency ("radiative equilibrium"). However, for relativistic systems, Boyer has suggested recently that the MB and RJ distributions might be incompatible. We have shown in Ref. 1, as mentioned above, that this is not the case.

In order to give a complete proof of the existence of an equilibrium state, it is necessary to show that not only does global equilibrium exist, but so does radiative equilibrium, that is, equilibrium at each frequency. This guarantees that the spectrum of the radiation does not change with time. The proof of the radiative equilibrium is the main purpose of this work. It is necessary to point out that we are considering the equilibrium between radia- tion and matter at a classical level. Therefore, we consider an isolated cavity with perfectly reflecting walls and a material system inside. With these conditions, the radiative equilibrium is equivalent to Kirchhoff's law, which is a well-known consequence of the general principles of thermodynamics. However, it has been conjectured ${ }^{6,7}$ that the Kirchhoff law might not be valid if one assumes the existence of a nonthermal zero-point field such that any wall would be partially transparent to it.

The plan of the paper is as follows. In Sec. II, we shall calculate the emitted power per unit frequency interval and in Sec. III, the absorbed power. Everywhere we follow the notations and the techniques of Ref. 1 . The calculation of the absorbed power rests upon a method which is explained in Appendix A. In Sec. IV, we discuss the results obtained. Finally, in Appendix B we discuss the relation between the emitted and absorbed power at every frequency and the reduced Fokker-Planck equation.

\section{EMITTED POWER}

As in Ref. 1, we consider a particle which, if unperturbed, would describe a multiply periodic motion. That motion is actually perturbed by the action of the random radiation and by the radiation reaction. However, we assume that these perturbations are small, in the sense that their effect in a deterministic period is negligible, so that, to lowest order, the power emitted can be calculated from the deterministic (i.e., unperturbed) motion averaged over all orbits with a suitable distribution function $W_{0}$ in phase space.

Now, according to Ref. 1, for a multiply periodic system immersed in a Rayleigh-Jeans radiation, $W_{0}$ depends to lowest order only on the energy, and then on $\overrightarrow{\mathbf{J}}$. Also, for multiply periodic motions the ensemble average over an orbit equals the time average ${ }^{1,8}$ which we must use in order to decouple the Fourier components of the motion. If we represent by $\zeta$ a point in phase space and by $P_{e}(\xi)$ the power emitted from that point, the total power emitted is

$$
\begin{aligned}
\int d \zeta W_{0}[\mathscr{E}(\overrightarrow{\mathbf{J}})] P_{e}(\zeta) & =\int d \overrightarrow{\mathbf{J}} d \overrightarrow{\mathbf{J}}^{\prime} d \overrightarrow{\mathrm{w}}^{\prime} W_{0}[\mathscr{E}(\overrightarrow{\mathbf{J}})] \int d \overrightarrow{\mathrm{w}} P_{e}\left(\overrightarrow{\mathrm{w}}, \overrightarrow{\mathbf{J}}, \overrightarrow{\mathrm{w}}^{\prime}, \overrightarrow{\mathbf{J}}^{\prime}\right) \\
& =\lim _{T \rightarrow \infty}(2 \pi)^{M} \int d \overrightarrow{\mathbf{J}} d \overrightarrow{\mathbf{J}}^{\prime} d \overrightarrow{\mathrm{w}}^{\prime} W_{0}[\mathscr{E}(\overrightarrow{\mathbf{J}})] \frac{1}{T} \int_{0}^{T} d t P_{e}\left(\overrightarrow{\mathrm{w}}(t), \overrightarrow{\mathbf{J}}_{,}, \overrightarrow{\mathrm{w}}^{\prime}, \overrightarrow{\mathrm{J}}^{\prime}\right)
\end{aligned}
$$


Due to the fact that both the result of the time average and $W_{0}$ do not depend on $\overrightarrow{\mathrm{w}}$, we can write $(2.1)$ as

$$
\begin{aligned}
\int d \zeta W_{0}[\mathscr{E}(\overrightarrow{\mathrm{J}})] P_{e}(\zeta) \\
\quad=\int d \zeta W_{0}[\mathscr{E}(\overrightarrow{\mathrm{J}})] \lim _{T \rightarrow \infty} \frac{1}{T} \int_{0}^{T} d t P_{e} \\
\quad=\int d \xi W_{0}[\mathscr{E}(\overrightarrow{\mathrm{J}})] \bar{P}_{e} .
\end{aligned}
$$

Therefore, the average in phase space can be put as a time average over each orbit ensemble averaged over phase space.

The emitted power per unit frequency interval is given by the energy carried per unit time by the Fourier components of the field created by the particle with frequencies between $\omega$ and $\omega+d \omega$. In order to calculate it, we take a sphere $\Sigma$ centered on the origin of coordinate $O$, that we take in such a way that the deterministic motion develops around it. The power emitted is given by

$$
P_{e}=\lim _{r \rightarrow \infty} \int_{\Sigma} d \vec{\sigma} \cdot \overrightarrow{\mathrm{S}}=\lim _{r \rightarrow \infty} r^{2} \int d \Omega \hat{r}^{0} \cdot \overrightarrow{\mathrm{S}},
$$

where $r$ is the radius of the sphere $\Sigma$ and $\vec{S}$ is the Poynting vector.

Taking into account that the contribution to radiation comes only from the acceleration fields ${ }^{9}$ and considering the time average over each orbit, we have

$\bar{P}_{e}=\lim _{T \rightarrow \infty} \frac{1}{T} \int_{0}^{T} d t \lim _{r \rightarrow \infty} \frac{c r^{2}}{4 \pi} \int d \Omega \hat{r}^{0} \cdot\left(\overrightarrow{\mathrm{E}}_{a} \times \overrightarrow{\mathrm{B}}_{a}\right)$,

where

$$
\begin{aligned}
& \overrightarrow{\mathrm{E}}_{a}=\left.\frac{e}{c} \frac{\hat{n} \times[(\hat{n}-\vec{\beta}) \times \dot{\vec{\beta}}]}{R(1-\vec{\beta} \cdot \hat{n})^{3}}\right|_{\mathrm{ret}}, \\
& \overrightarrow{\mathbf{B}}_{a}=\left.\hat{n}\right|_{\mathrm{ret}} \times \overrightarrow{\mathrm{E}}_{a}, \\
& \hat{n}=\frac{\overrightarrow{\mathbf{r}}-\vec{\xi}(t)}{|\overrightarrow{\mathrm{r}}-\vec{\xi}(t)|}, \\
& R=|\overrightarrow{\mathrm{r}}-\vec{\xi}(t)|,
\end{aligned}
$$

and the subscript ret means that the corresponding quantity must be calculated at the retarded time.

From (2.5a) and (2.5b), it is seen that

$$
\begin{aligned}
\overrightarrow{\mathrm{E}}_{a} \times \overrightarrow{\mathrm{B}}_{a} & =\overrightarrow{\mathrm{E}}_{a} \times\left(\left.\hat{n}\right|_{\text {ret }} \times \overrightarrow{\mathrm{E}}_{a}\right) \\
& =\left.E_{a}{ }^{2} \hat{n}\right|_{\text {ret }}-\left.\overrightarrow{\mathrm{E}}_{a} \cdot \hat{n}\right|_{\text {ret }} \overrightarrow{\mathrm{E}}_{a}=\left.E_{a}{ }^{2} \hat{n}\right|_{\text {ret }},
\end{aligned}
$$

whence it follows

$\bar{P}_{e}=\left.\lim _{T \rightarrow \infty} \frac{1}{T} \int_{0}^{T} d t \lim _{r \rightarrow \infty} \frac{c}{4 \pi} r^{2} \int d \Omega \hat{r}^{0} \cdot \hat{n}\right|_{\mathrm{ret}} E_{a}{ }^{2}$.

Now, in the limit $r \rightarrow \infty$, we have

$$
\hat{n}=\hat{r}^{0}+\vec{\delta}, \quad \vec{\delta}=O\left(\left|\frac{\xi}{r}\right|\right)
$$

with

$$
\vec{\delta} \cdot \hat{r}^{0}=0
$$

so that

$$
\hat{n} \cdot \hat{r}^{0}=1+\delta^{\prime}, \quad \delta^{\prime}=O\left(\left|\frac{\xi}{r}\right|^{2}\right),
$$

and then

$$
\begin{aligned}
\bar{P}_{e}= & \lim _{T \rightarrow \infty} \frac{1}{T} \int_{0}^{T} d t \lim _{r \rightarrow \infty} \frac{c}{4 \pi} r^{2} \int d \Omega E_{a}{ }^{2} \\
& +\lim _{T \rightarrow \infty} \frac{1}{T} \int_{0}^{T} d t \lim _{r \rightarrow \infty} \frac{c}{4 \pi} r^{2} \int d \Omega \delta^{\prime} E_{a}{ }^{2} .
\end{aligned}
$$

The second integral is of order $1 / r^{2}$ because in a multiply periodic motion the acceleration and the velocity are bounded, and the $R E_{a}$ is also bounded. Therefore that integral goes to zero in the limit $r \rightarrow \infty$. Consequently

$$
\bar{P}_{e}=\lim _{T \rightarrow \infty} \frac{1}{T} \int_{0}^{T} d t \lim _{r \rightarrow \infty} \frac{c}{4 \pi} r^{2} \int d \Omega E_{a}^{2} .
$$

A procedure similar to that used in Ref. 1 with $\overrightarrow{\mathrm{h}}_{\varphi}$ and $\vec{g}$ shows that $\overrightarrow{\mathrm{E}}_{a}$ can be expanded in the form

$$
\overrightarrow{\mathrm{E}}_{a}=\sum_{\overrightarrow{\mathrm{n}}} \overrightarrow{\mathrm{E}}_{\overrightarrow{\mathrm{n}}} e^{i \overrightarrow{\mathrm{n}} \cdot \vec{\omega}_{0} t}
$$

which together with (2.10) gives

$$
\begin{aligned}
\bar{P}_{e}= & \lim _{T \rightarrow \infty} \lim _{r \rightarrow \infty} \frac{1}{T} \int_{0}^{T} d t \frac{c}{4 \pi} r^{2} \\
& \quad \times \int d \Omega \sum_{\overrightarrow{\mathrm{n}}} \sum_{\overrightarrow{\mathrm{m}}} \overrightarrow{\mathrm{E}}_{\overrightarrow{\mathrm{n}}} \cdot \overrightarrow{\mathrm{E}}_{\overrightarrow{\mathrm{m}}} e^{i(\overrightarrow{\mathrm{n}}+\overrightarrow{\mathrm{m}}) \cdot \vec{\omega}_{0} t} \\
= & \lim _{r \rightarrow \infty} \frac{c}{4 \pi} r^{2} \int d \Omega \sum_{\overrightarrow{\mathrm{n}}} \overrightarrow{\mathrm{E}}_{\overrightarrow{\mathrm{n}}} \cdot \overrightarrow{\mathrm{E}}_{-\overrightarrow{\mathrm{n}}}
\end{aligned}
$$

As $\overrightarrow{\mathrm{E}}$ is real, $\overrightarrow{\mathrm{E}}_{-\overrightarrow{\mathrm{n}}}=\overrightarrow{\mathrm{E}}_{\overrightarrow{\mathrm{n}}}^{*}$ and therefore

$$
\bar{P}_{e}=\lim _{r \rightarrow \infty} \frac{c}{4 \pi} r^{2} \int d \Omega \sum_{\overrightarrow{\mathrm{n}}}\left|\overrightarrow{\mathrm{E}}_{\overrightarrow{\mathrm{n}}}\right|^{2}=\sum_{\overrightarrow{\mathrm{n}}} \bar{P}_{e}\left(\overrightarrow{\mathrm{n}} \cdot \vec{\omega}_{0}\right)
$$

with

$$
\bar{P}_{e}\left(\overrightarrow{\mathrm{n}} \cdot \vec{\omega}_{0}\right)=\lim _{r \rightarrow \infty} \frac{c}{4 \pi} r^{2} \int d \Omega\left|\overrightarrow{\mathrm{E}}_{\overrightarrow{\mathrm{n}}}\right|^{2}
$$

The meaning of these quantities can be easily understood. If the acceleration field of the particle includes only one frequency, i.e.,

$$
\overrightarrow{\mathrm{E}}_{a}\left(\overrightarrow{\mathrm{n}}_{0}\right)=\overrightarrow{\mathrm{E}}_{0} e^{i \overrightarrow{\mathrm{n}}_{0} \cdot \vec{\omega}_{0} t}+\overrightarrow{\mathrm{E}}_{0}^{*} e^{-i \overrightarrow{\mathrm{n}}_{0} \cdot \vec{\omega}_{0} t},
$$

the corresponding average emitted power is given by

$$
\begin{aligned}
\bar{P}_{e}\left(\overrightarrow{\mathrm{n}}_{0}\right) & =\lim _{T \rightarrow \infty} \frac{1}{T} \int_{0}^{T} d t \lim _{r \rightarrow \infty} \frac{c}{4 \pi} r^{2} \int d \Omega\left|\overrightarrow{\mathrm{E}}_{a}\left(\overrightarrow{\mathrm{n}}_{0}\right)\right|^{2} \\
& =\bar{P}_{e}\left(\overrightarrow{\mathrm{n}}_{0} \cdot \vec{\omega}_{0}\right)+\bar{P}_{e}\left(-\overrightarrow{\mathrm{n}}_{0} \cdot \vec{\omega}_{0}\right)
\end{aligned}
$$

In view of that, the emitted power at a frequency $\omega$, $I_{e}(\omega)$ can be written, after averaging over phase space,

$$
\begin{aligned}
I_{e}(\omega)=\int & d \zeta W_{0}(\xi) \\
& \times \sum_{\overrightarrow{\mathrm{n}}}^{\prime} \delta\left(\omega-\overrightarrow{\mathrm{n}} \cdot \vec{\omega}_{0}\right)\left[\bar{P}_{e}\left(\overrightarrow{\mathrm{n}} \cdot \vec{\omega}_{0}\right)+\bar{P}_{e}\left(-\overrightarrow{\mathbf{n}} \cdot \vec{\omega}_{0}\right)\right],
\end{aligned}
$$


where $\Sigma^{\prime}$ is the sum extended to those values of $\vec{n}$ such that

$$
\overrightarrow{\mathrm{n}} \cdot \vec{\omega}_{0}=\sum_{i=1}^{M} n_{i} \omega_{i}>0
$$

Let us calculate the term $\bar{P}_{e}\left(\vec{n} \cdot \vec{\omega}_{0}\right)$ given by (2.14). To do that, we must calculate the Fourier coefficients of the electric field $\overrightarrow{\mathrm{E}}(t)$

$$
\overrightarrow{\mathbf{E}}_{\overrightarrow{\mathbf{n}}}=\lim _{T \rightarrow \infty} \frac{1}{T} \int_{0}^{T} d t \overrightarrow{\mathrm{E}}_{a}(t) e^{-i \overrightarrow{\mathrm{n}} \cdot \vec{\omega}_{0} t}=\left.\lim _{T \rightarrow \infty} \frac{1}{T} \int_{0}^{T} d t \frac{e}{c}\left[\frac{\hat{n} \times[(\hat{n}-\vec{\beta}) \times \dot{\vec{\beta}}]}{R(1-\vec{\beta} \cdot \hat{n})^{3}}\right]\right|_{\mathrm{ret}} e^{-i \overrightarrow{\mathrm{n}} \cdot \vec{\omega}_{0} t}
$$

As is usual (see Ref. 1), we make the change in the integral $t \rightarrow t^{\prime}=t_{\text {ret }}(t, \overrightarrow{\mathrm{r}})$. With the reasoning of Ref. 1 we write

$$
\overrightarrow{\mathrm{E}}_{\overrightarrow{\mathrm{n}}}=\lim _{T \rightarrow \infty} \frac{1}{T} \int_{0}^{T} d t^{\prime} \frac{e}{c} \frac{\hat{n} \times[(\hat{n}-\vec{\beta}) \times \dot{\vec{\beta}}]}{R(1-\vec{\beta} \cdot \hat{n})^{3}} e^{-i \overrightarrow{\mathrm{n}} \cdot \vec{\omega}_{0} t\left(t^{\prime}\right)} \frac{d t}{d t^{\prime}}
$$

and

$$
\begin{aligned}
& t=t^{\prime}+\frac{1}{c}\left|\overrightarrow{\mathrm{r}}-\vec{\xi}\left(t^{\prime}\right)\right|=t^{\prime}+\frac{1}{c} R, \\
& \frac{d t}{d t^{\prime}}=1-\vec{\beta} \cdot \hat{n} .
\end{aligned}
$$

Now, in the limit $r \rightarrow \infty, R \cong r-\hat{r}^{0} \cdot \vec{\xi}$ and $\hat{n}=\hat{r}^{0}+\vec{\delta}$ with $\vec{\delta}$ given by $(2.8 \mathrm{a})$, whence we have, neglecting terms in $1 / r$,

$$
\overrightarrow{\mathrm{E}}_{\overrightarrow{\mathrm{n}}}=\lim _{T \rightarrow \infty} \frac{1}{T} \int_{0}^{T} d t^{\prime} \frac{e}{c} \frac{\hat{r}^{0} \times\left[\left(\hat{r}^{0}-\vec{\beta}\right) \times \dot{\vec{\beta}}\right]}{r\left(1-\vec{\beta} \cdot \hat{r}^{0}\right)^{2}} e^{-i \overrightarrow{\mathrm{n}} \cdot \vec{\omega}_{0} t^{\prime}-i \overrightarrow{\mathrm{n}} \cdot \vec{\omega}_{0} r / c} e^{i \overrightarrow{\mathrm{n}} \cdot \vec{\omega}_{0}\left(\hat{r}^{0} \cdot \vec{\xi} / c\right)} .
$$

It is trivial to show that

$$
\frac{\hat{r}^{0} \times\left[\left(\hat{r}^{0}-\vec{\beta}\right) \times \dot{\vec{\beta}}\right]}{\left(1-\vec{\beta} \cdot \hat{r}^{0}\right)^{2}}=\frac{d}{d t}\left[\frac{\hat{r}^{0} \times\left(\hat{r}^{0} \times \vec{\beta}\right)}{1-\vec{\beta} \cdot \hat{r}^{0}}\right),
$$

whence we obtain, after an integration by parts,

$$
\overrightarrow{\mathrm{E}}_{\overrightarrow{\mathrm{n}}}=\lim _{T \rightarrow \infty} \frac{1}{T} \int_{0}^{T} d t^{\prime} \frac{e}{c}\left[\hat{r}^{0} \times\left(\hat{r}^{0} \times \vec{\beta}\right)\right]\left(t^{\prime}\right) i \overrightarrow{\mathrm{n}} \cdot \vec{\omega}_{0} e^{-i \overrightarrow{\mathrm{n}} \cdot \vec{\omega}_{0} t^{\prime}-i \overrightarrow{\mathrm{n}} \cdot \vec{\omega}_{0} r / c} e^{i \overrightarrow{\mathrm{n}} \cdot \vec{\omega}_{0}\left(\hat{r}^{0} \cdot \vec{\xi}\right) / c}
$$

and, finally, it results in

$$
\begin{aligned}
& \bar{P}_{e}\left(\overrightarrow{\mathbf{n}} \cdot \vec{\omega}_{0}\right)=\lim _{r \rightarrow \infty} \frac{c}{4 \pi} r^{2} \int d \Omega \lim _{T \rightarrow \infty} \lim _{T^{\prime} \rightarrow \infty} \frac{1}{T T^{\prime}} \int_{0}^{T} d t_{1} \int_{0}^{T^{\prime}} d t_{2}\left[-\frac{e^{2}}{c^{2}}\right]\left(\overrightarrow{\mathbf{n}} \cdot \vec{\omega}_{0}\right)^{2} \\
& \\
& \times\left(\left\{\hat{r}^{0} \times\left[\hat{r}^{0} \times \vec{\beta}\left(t_{1}\right)\right]\right\} \cdot\left\{\hat{r}^{0} \times\left[\hat{r}^{0} \times \vec{\beta}\left(t_{2}\right)\right]\right\}\right) e^{-i \overrightarrow{\mathbf{n}} \cdot \vec{\omega}_{0}\left(t_{1}-t_{2}\right)} \\
& \times \exp \left[i \overrightarrow{\mathbf{n}} \cdot \vec{\omega}_{0}\left[\frac{\hat{r}^{0} \cdot\left[\vec{\xi}\left(t_{1}\right)-\vec{\xi}\left(t_{2}\right)\right]}{c}\right]\right] .
\end{aligned}
$$

The product between heavy parentheses can be written

$$
\vec{\beta}\left(t_{1}\right) \cdot\left(I-\hat{r}^{0} \hat{r}^{0}\right) \cdot \vec{\beta}\left(t_{2}\right)
$$

with the notation of Ref. 1. With the change $t_{1}-t_{2}=u$ and using (2.9) of that reference we get

$\bar{P}_{e}\left(\overrightarrow{\mathrm{n}} \cdot \vec{\omega}_{0}\right)=-\frac{e^{2}}{4 \pi c} \frac{\left(\overrightarrow{\mathrm{n}} \cdot \vec{\omega}_{0}\right)^{2}}{(2 \pi)^{M}} \int d \overrightarrow{\mathrm{w}} \lim _{T \rightarrow \infty} \frac{1}{T} \int_{0}^{T} d u e^{-i \overrightarrow{\mathrm{n}} \cdot \vec{\omega}_{0} u} \int d \Omega \vec{\beta} \cdot\left(I-\hat{r}^{0} \hat{r}^{0}\right) \cdot \vec{\beta}-u \exp \left[i \overrightarrow{\mathrm{n}} \cdot \vec{\omega}_{0}\left[\frac{\hat{r}^{0} \cdot\left(\overrightarrow{\mathrm{r}}-\overrightarrow{\mathrm{r}}^{-u}\right)}{c}\right]\right]$.

Taking into account Eq. (5.22) of Ref. 1, we have

$\sigma[\mathscr{E}]\left(\omega, \overrightarrow{\mathrm{w}}, u ; \vec{\omega}_{0}\right)=c \int d \Omega \exp \left[i \frac{\omega}{c} \hat{r}^{0} \cdot\left(\overrightarrow{\mathrm{r}}-\overrightarrow{\mathrm{r}}^{-u}\right)\right] \vec{\beta} \cdot\left(I-\hat{r}^{0} \hat{r}^{0}\right) \cdot \vec{\beta}-u=\sum_{\overrightarrow{\mathrm{n}}, \overrightarrow{\mathrm{m}}} \sigma_{\overrightarrow{\mathrm{n}}, \overrightarrow{\mathrm{m}}}[\mathscr{E}]\left(\omega, \vec{\omega}_{0}\right) e^{i \overrightarrow{\mathrm{n}} \cdot \overrightarrow{\mathrm{w}}} e^{-i \overrightarrow{\mathrm{m}} \cdot \vec{\omega}_{0}^{u}}$,

and therefore 


$$
\begin{aligned}
\bar{P}_{e}\left(\overrightarrow{\mathrm{n}} \cdot \vec{\omega}_{0}\right) & =-\frac{e^{2}}{4 \pi c} \frac{\left(\overrightarrow{\mathrm{n}} \cdot \vec{\omega}_{0}\right)^{2}}{(2 \pi)^{M}} \int d \overrightarrow{\mathrm{w}} \lim _{T \rightarrow \infty} \frac{1}{T} \int_{0}^{T} d u e^{-i \overrightarrow{\mathrm{n}} \cdot \vec{\omega}_{0} u} \frac{1}{c} \sigma[\mathscr{E}]\left(\overrightarrow{\mathrm{n}} \cdot \vec{\omega}_{0}, \overrightarrow{\mathrm{w}}, u ; \vec{\omega}_{0}\right) \\
& =-\frac{e^{2}}{4 \pi c^{2}} \frac{\left(\overrightarrow{\mathrm{n}} \cdot \vec{\omega}_{0}\right)^{2}}{(2 \pi)^{M}} \int d \overrightarrow{\mathrm{w}} \lim _{T \rightarrow \infty} \frac{1}{T} \int_{0}^{T} d u e^{-i \overrightarrow{\mathrm{n}} \cdot \vec{\omega}_{0} u} \sum_{\overrightarrow{\mathrm{K}}, \overrightarrow{\mathrm{m}}} \sigma_{\overrightarrow{\mathrm{K}}, \overrightarrow{\mathrm{m}}}[\mathscr{E}]\left(\overrightarrow{\mathrm{n}} \cdot \vec{\omega}_{0} ; \vec{\omega}_{0}\right) e^{i \overrightarrow{\mathrm{K}} \cdot \overrightarrow{\mathrm{w}}} e^{-i \overrightarrow{\mathrm{m}} \cdot \vec{\omega}_{0} u}
\end{aligned}
$$

Hence

$$
\overline{\boldsymbol{P}}_{a}\left(\overrightarrow{\mathrm{n}} \cdot \vec{\omega}_{0}\right)=-\frac{e^{2}}{4 \pi c^{2}}\left(\overrightarrow{\mathrm{n}} \cdot \vec{\omega}_{0}\right)^{2} \sigma_{\overrightarrow{0},-\overrightarrow{\mathrm{n}}}[\mathscr{E}]\left(\overrightarrow{\mathrm{n}} \cdot \vec{\omega}_{0} ; \vec{\omega}_{0}\right)
$$

Finally, from (2.26) and (2.15),

$$
I_{e}(\omega)=-\frac{e^{2}}{2 \pi c^{2}} \omega^{2}(2 \pi)^{M} \int d \overrightarrow{\mathbf{J}} d \overrightarrow{\mathbf{J}}^{\prime} d \overrightarrow{\mathrm{w}}^{\prime} W_{0}[\mathscr{E}(\overrightarrow{\mathbf{J}})] \sum_{\overrightarrow{\mathbf{n}}}^{\prime} \delta\left(\omega-\overrightarrow{\mathbf{n}} \cdot \vec{\omega}_{0}\right) \operatorname{Re}\left\{\sigma_{\overrightarrow{0},-\overrightarrow{\mathbf{n}}}[\mathscr{E}]\left(\overrightarrow{\mathbf{n}} \cdot \vec{\omega}_{0} ; \vec{\omega}_{0}\right)\right\}
$$

where we have used

$$
\sigma_{\overrightarrow{\mathbf{m}}, \overrightarrow{\mathbf{n}}}[\varphi]\left(\omega ; \vec{\omega}_{0}\right)=\sigma_{-\overrightarrow{\mathbf{m}},-\overrightarrow{\mathbf{n}}}^{*}[\varphi]\left(-\omega ; \vec{\omega}_{0}\right),
$$

and the fact that the term under the integral sign does not depend on $\vec{w}$.

\section{ABSORBED POWER}

We must obtain the energy absorbed by the particle from the background field. As the magnetic field does not produce work, we should consider only the electric field. We must obtain the absorbed power by the particle from the component $\overrightarrow{\mathrm{E}}_{\omega, \Delta \omega}(\overrightarrow{\mathrm{r}}, t)$ of the field whose angular frequency is between $\omega$ and $\omega+\Delta \omega$. This can be written (see Ref. 1)

$$
\begin{aligned}
& \overrightarrow{\mathrm{E}}_{\omega, \Delta \omega}(\overrightarrow{\mathrm{r}}, t)=\int_{\omega}^{\omega+\Delta \omega} d \omega^{\prime} \frac{\omega^{\prime 2}}{c^{3}} \mathscr{Y}\left(\omega^{\prime}\right) \\
& \times \sum_{\lambda=1}^{2} \int d \Omega_{\overrightarrow{\mathrm{k}}} \vec{\epsilon}(\overrightarrow{\mathrm{k}}, \lambda) \\
& \\
& \quad \times \cos \left[\overrightarrow{\mathrm{k}} \cdot \overrightarrow{\mathrm{r}}-\omega^{\prime} t+\theta(\overrightarrow{\mathrm{k}}, \lambda)\right],
\end{aligned}
$$

where $\theta$ is a random phase with uniform distribution. If we consider a given sample of the background stochastic field, we assume that the state of the system is given at time $t$ by the phase-space distribution $\rho(\xi, t)$ so that the absorbed power from $\overrightarrow{\mathrm{E}}_{\omega, \Delta \omega}$ is given by

$$
P_{a}(\omega, \omega+\Delta \omega, t)=\int d \zeta \overrightarrow{\mathrm{V}} \cdot e \overrightarrow{\mathrm{E}}_{\omega, \Delta \omega}(\zeta, t) \rho(\zeta, t) .
$$

If we average to all samples of the stochastic field we have

$$
\left\langle\boldsymbol{P}_{a}(\omega, \omega+\Delta \omega, t)\right\rangle=\int d \xi e \overrightarrow{\mathrm{V}} \cdot\left\langle\overrightarrow{\mathrm{E}}_{\omega, \Delta \omega}(\xi, t) \rho(\zeta, t)\right\rangle .
$$

The absorbed power at the stationary state will be obtained taking the limit

$$
\begin{aligned}
P_{a}(\omega, \omega+\Delta \omega) & =\lim _{t \rightarrow \infty}\left\langle P_{a}(\omega, \omega+\Delta \omega, t)\right\rangle \\
& =\lim _{t \rightarrow \infty} \int d \zeta e \overrightarrow{\mathrm{V}} \cdot\left\langle\overrightarrow{\mathrm{E}}_{\omega, \Delta \omega}(\zeta, t) \rho(\zeta, t)\right\rangle .
\end{aligned}
$$

If we write the probability density as a sum of the equilibrium density $W_{0}$-uncorrelated with the fieldplus fluctuations $\delta \rho$, we see that the absorption of energy is due to the correlation between the stochastic field and the density fluctuations.

In our problem, the stochastic force is not a white noise and the correlations must be calculated with some approximation procedure. Problems of this type are usually solved using $\mathrm{Kubo}^{10}$ linear response theory which, in fact, has been applied to the nonrelativistic theory of radiation-matter equilibrium. ${ }^{4}$ Kubo theory allows the calculation of the power absorbed by a system in statistical equilibrium from an external electromagnetic field up to lowest order. It is assumed that statistical equilibrium exists before the external field is applied. However, in our case this condition is not fulfilled because the equilibrium is produced by the same external field (the background radiation) from which the absorption must be calculated. Therefore it should be necessary to perform a dynamical calculation of the correlation between $\overrightarrow{\mathrm{E}}_{\omega, \Delta \omega}$ and $\rho$. In Appendix A, we develop this calculation by a method which allows to obtain the correlation from a series of cumulants which converges asymptotically. The final result can be written

$$
\begin{aligned}
\lim _{t \rightarrow \infty}\left\langle e\left(E_{\omega, \Delta \omega}(\xi, t)\right)_{i} \rho(\xi, t)\right\rangle=-\int_{\omega}^{\omega+\Delta \omega} d \omega^{\prime} \frac{e^{2} \mathscr{Y}^{2}\left(\omega^{\prime}\right)}{2 c^{3}} \omega^{\prime 2} \sum_{j=1}^{3} \int_{0}^{\infty} d u \int d \Omega T_{i j}\left(\overrightarrow{\mathrm{V}}^{-u}\right) & \times \cos \left[\omega^{\prime} \frac{\hat{r}^{0}}{c} \cdot\left(\overrightarrow{\mathrm{r}}-\overrightarrow{\mathrm{r}}^{-u}\right)-\omega^{\prime} u\right] \frac{\partial}{\partial p_{j}^{-u}} W_{0}(\zeta)
\end{aligned}
$$


where $T_{i j}$ is given by (A40).

If the spectrum of the radiation is of the RJ type-i.e., $\mathscr{Y}^{2}=$ constant- $W_{0}$ is to lowest order a function of the deterministic energy alone. Then

$$
\frac{\partial}{\partial p_{j}^{-u}} W_{0}=V_{j}^{-u} W_{0}^{\prime}[\mathscr{E}]
$$

Therefore

$$
\sum_{j=1}^{3} T_{i j}\left(\overrightarrow{\mathrm{V}}^{-u}\right) \frac{\partial}{\partial p_{j}^{-u}} W_{0}(\xi)=\left(I-\hat{r}^{0} \hat{r}^{0}\right) \cdot \overrightarrow{\mathrm{V}}^{-u}
$$

whence it follows

$$
\lim _{t \rightarrow \infty}\left\langle e \overrightarrow{\mathrm{E}}_{\omega, \Delta \omega}(\zeta, t) \rho(\zeta, t)\right\rangle=-\frac{e^{2} \mathscr{Y}^{2}}{c^{3}} \int_{\omega}^{\omega+\Delta \omega} d \omega^{\prime} \omega^{\prime 2} \int_{0}^{\infty} d u \int d \Omega\left(I-\hat{r}^{0} \hat{r}^{0}\right) V^{-u} \cos \left[\frac{\omega^{\prime}}{c} \hat{r}^{0} \cdot\left(\overrightarrow{\mathrm{r}}-\overrightarrow{\mathrm{r}}^{-u}\right)-\omega^{\prime} u\right] W_{0}^{\prime}
$$

and the absorbed power per unit frequency is

$I_{a}(\omega)=\lim _{\Delta \omega \rightarrow 0} \frac{P_{a}(\omega, \omega+\Delta \omega)}{\Delta \omega}=-e^{2} \frac{\mathscr{Y}^{2} \omega^{2}}{c^{3}} \int d \zeta \int_{0}^{\infty} d u \int d \Omega \overrightarrow{\mathrm{V}} \cdot\left(I-\hat{r}^{0} \hat{r}^{0}\right) \cdot \overrightarrow{\mathrm{V}}-u \cos \left[\frac{\omega}{c} \hat{r}^{0}\left(\overrightarrow{\mathbf{r}}-\overrightarrow{\mathbf{r}}^{-u}\right)-\omega u\right] W_{0}^{\prime}$

It is possible to extend the integral over $u$ to negative values. To do that we consider Eq. (3.9) for $u<0$,

$$
R=-e^{2} \mathscr{Y}^{2} \frac{\omega^{2}}{c^{3}} \int d \xi \int_{-\infty}^{0} d u \int d \Omega \overrightarrow{\mathrm{V}} \cdot\left(I-\hat{r}^{0} \hat{r}^{0}\right) \cdot \overrightarrow{\mathrm{V}}-u \cos \left(\frac{\omega}{c} \hat{r}^{0}\left(\overrightarrow{\mathrm{r}}-\overrightarrow{\mathrm{r}}^{-u}\right)-\omega u\right) W_{0}^{\prime} .
$$

Then, we can change $u^{\prime}=-u$ and $\zeta^{\prime}=\zeta^{u^{\prime}}$, so that $d \xi=d \zeta^{\prime}$ and $\zeta=\xi^{\prime}-u^{\prime}$ with the result that Eq. (3.10) agrees with (3.9).

Therefore, we can write

$$
I_{a}(\omega)=-\frac{e^{2} \omega^{2} \mathscr{Y}^{2}}{2 c^{3}} \int d \xi \int_{-\infty}^{+\infty} d u \int d \Omega \overrightarrow{\mathrm{V}} \cdot\left(I-\hat{r}^{0} \hat{r}^{0}\right) \cdot \overrightarrow{\mathrm{V}}-u \cos \left[\frac{\omega}{c} \hat{r}^{0} \cdot\left(\overrightarrow{\mathrm{r}}-\overrightarrow{\mathrm{r}}^{-u}\right)-\omega u\right] W_{0}^{\prime}
$$

Using again (2.24) we obtain

$$
\begin{aligned}
I_{a}(\omega) & =-e^{2} \frac{\omega^{2} \mathscr{Y}^{2}}{4 c^{2}} \int d \zeta \int_{-\infty}^{+\infty} d u W_{0}^{\prime} \operatorname{Re}\left\{\sigma[\mathscr{E}]\left(\omega, \overrightarrow{\mathrm{w}}, u ; \vec{\omega}_{0}\right) e^{-i \omega u}\right\} \\
& =-e^{2} \frac{\omega^{2} \mathscr{Y}^{2}}{4 c^{2}} \operatorname{Re}\left[\int d \overrightarrow{\mathrm{J}} d \overrightarrow{\mathrm{J}}{ }^{\prime} d \overrightarrow{\mathrm{w}}^{\prime} W_{0}^{\prime} \int d \overrightarrow{\mathrm{w}} \int_{-\infty}^{+\infty} d u \sum_{\overrightarrow{\mathrm{m}}, \overrightarrow{\mathrm{n}}} \sigma_{\overrightarrow{\mathrm{m}}, \overrightarrow{\mathrm{n}}}[\mathscr{E}]\left(\omega ; \vec{\omega}_{0}\right) e^{i \overrightarrow{\mathrm{m}} \cdot \overrightarrow{\mathrm{w}}} e^{-i \overrightarrow{\mathrm{n}} \cdot \overrightarrow{\omega_{0}} u} e^{-i \omega u}\right]
\end{aligned}
$$

and, finally

$$
I_{a}(\omega)=-\frac{e^{2} \omega^{2} \mathscr{Y}^{2}}{2 c^{2}}(2 \pi)^{M} \pi \sum_{\overrightarrow{\mathbf{n}}} \sum^{\prime} \int d \overrightarrow{\mathbf{J}} d \overrightarrow{\mathbf{J}}{ }^{\prime} d \overrightarrow{\mathbf{w}}^{\prime} W_{0}^{\prime}[\mathscr{E}(\overrightarrow{\mathbf{J}})] \delta\left(\omega-\overrightarrow{\mathbf{n}} \cdot \vec{\omega}_{0}\right) \operatorname{Re}\left\{\sigma_{\overrightarrow{0},-\overrightarrow{\mathbf{n}}}[\mathscr{E}]\left(\omega ; \vec{\omega}_{0}\right)\right\}
$$

Taking into account that, according to Ref. 1,

$$
W_{0}^{\prime}=-\frac{1}{\pi^{2} \mathscr{Y}^{2}} W_{0},
$$

we have the desired result

$$
I_{e}(\omega)+I_{a}(\omega)=0 .
$$

We shall study in Appendix B the relation between the emitted and the absorbed power per unit frequency, and the coefficients of the reduced Fokker-Planck equation of Ref. 1. Moreover, we shall show that in the case of the Rayleigh-Jeans spectrum a stronger type of balance which implies not only radiative balance (3.14), but also detailed balance, is satisfied.

\section{DISCUSSION}

The conclusion of our work (including Ref. 1) is twofold: First, that a relativistic material system immersed in a background radiation with a Rayleigh-Jeans spectrum approaches a stationary state given by the MaxwellBoltzmann distribution; ${ }^{1}$ secondly, in this paper we have proven that in this state the system is also in radiative equilibrium, which means that the energy of the system does not change on the average and that the spectrum of the radiation does not change either. In this paper we have not proven that it is not possible to have equilibrium with a radiation having a different spectrum. The proof of this statement can be done with a particular system showing that equilibrium does not exist if the radiation 
spectrum is different from the RJ one. Actually, this has been made with the nonrelativistic anharmonic oscillator. ${ }^{4}$

Our results show that there is no inconsistency between MB and RJ distributions, contrary to Boyer's conjecture, ${ }^{5}$ thus putting on a firm basis classical relativistic statistical mechanics. However, there is a difficulty with the fact that the RJ distribution is not a true spectrum, because it gives rise to a divergent energy density. We shall comment on this problem below, but before that we shall study the validity of the approximations involved in our study.

Obviously, the first limitation comes from the difficulties of the classical electrodynamics of point particles. In our work, we have considered all deterministic orbits, including the ones with an energy near the minimum of the potential (assuming that it exists). When the size of the orbit is of the order of the classical radius of the electron, the model of a point particle lacks its validity, i.e., the Lorentz-Dirac equation is no longer correct. In order that this difficulty may be overcome it is necessary that such orbits have a small probability. As the average energy of the MB distribution is $k \theta=\pi^{2} \mathscr{Y}^{2}$ ( $\theta$ being the absolute temperature), we see that our results will be not valid for too low temperatures. Of course, this problem is not specific of a relativistic theory.

The main approximation involved in the solution of the stochastic differential equations of motion is the Markovian one. ${ }^{11,12}$ This approximation rests upon the assumption that the forces due to the damping (radiation reaction) and the background field are small. More precisely, the following inequalities should hold:

$$
\begin{gathered}
\tau_{c} \ll \tau_{r}^{\mathrm{st}}, \\
\tau_{c} \ll \tau_{r}^{\mathrm{d}},
\end{gathered}
$$

where $\tau_{c}$ is the correlation time of the stochastic force, $\tau_{r}$ is the relaxation time, that is the time needed for the effect of the force to be non-negligible. Here st stands for stochastic and d for damping. Also, in order to use the Haken $^{13}$ reduction procedure, it is necessary for the deterministic time to be small in comparison with both relaxation times:

$$
\begin{aligned}
& T \ll \tau_{r}^{\mathrm{st}}, \\
& T \ll \tau_{r}^{\mathrm{d}} .
\end{aligned}
$$

The deterministic time $T$ is essentially the period of the deterministic motion. This condition is necessary in order that the orbit is not changed too much by the action of the stochastic and damping forces.

The first problem we have is that the Rayleigh-Jeans spectrum does not have a correlation time because it diverges at high frequencies (ultraviolet catastrophe). The problem of the divergences is a fundamental one of classical electrodynamics and we will not study it here in detail. A possible solution is to use extended-instead of pointlike-particles. The extended particle produces an effective cutoff in the frequencies of the stochastic force whose inverse can be taken as a correlation time. For some reasonable models of extended particles ${ }^{14}$ we have

$$
\tau_{c} \sim 10 \tau_{0}
$$

where

$$
\tau_{0}=\frac{2 e^{2}}{3 m c_{3}} .
$$

As we shall see later, an estimate of condition (4.2b) leads to the inequality $T \gg \tau_{c}$ for moderately relativistic temperatures, which guarantees that, under those conditions, inequality (4.1b) also holds.

Moreover, at the equilibrium state, both relaxation times $\tau_{r}^{\text {st }}$ and $\tau_{r}^{\mathrm{d}}$ should be of the same order. Therefore, the estimation of the condition $(4.2 b)$ alone is enough to guarantee the validity of the approximation.

It is to be noted that the spectrum of the background field appears multiplied by the absolute temperature $\theta$. As long as the spectrum gives the order of magnitude of the random force intensity, it seems reasonable to think that, if the temperature is too high, the effect of the random force will no longer be perturbative. An important question is whether or not the bound for $\theta$ allows for relativistic velocities, because, if it does, the results obtained will be valid, at least, for moderately relativistic systems.

What we have to verify is that inequality $(4.2 \mathrm{~b})$ holds for those orbits for which energies are of the order of the most probable value, that is, $k \theta$. Clearly, this condition depends on the deterministic force both because the radiation of energy depends on the acceleration of the charge, and because the average energy depends on the external field. Consequently, our estimation is not necessarily valid for all the systems.

First, we suppose that the averages of the kinetic energy, $E_{c}=m c^{2}(\gamma-1)$, and the total one are of the same order; and, secondly, we calculate the accelerations as if the orbits were circular. It is clear that only in each particular case can more precise estimates be made.

Condition (4.2b) means that the energy lost by the particle in a period of the motion is small in comparison with the total energy, i.e.,

$$
\left|P_{a}\right| T<<E \text {. }
$$

Now

$$
\left|P_{a}\right|=\frac{2 e^{2}}{3 c^{3}} \alpha_{\lambda} \alpha^{\lambda}
$$

and $\alpha_{\lambda} \alpha^{\lambda}$ has values between $\gamma^{4} a^{2}$ and $\gamma^{6} a^{2}$. Although for a circular orbit $\alpha^{\lambda} \alpha_{\lambda}$ takes the smallest of these values, we consider the largest one which improves the estimation. Then we have

$$
T \frac{2 e^{2}}{3 c^{3}} \gamma^{6} a^{2} \ll m c^{2}(\gamma-1) .
$$

As we said above, the acceleration can be written as

$$
a \sim v / T
$$

and then we have

$$
\frac{2 e^{2} v^{2}}{3 c^{3} T} \gamma^{6}<<m c^{2}(\gamma-1) .
$$

Writing

$$
\frac{k \theta}{m c^{2}}=\delta,
$$


we obtain

$$
\gamma=1+\delta, \quad v^{2} / c^{2}=\delta(\delta+2)(\delta+1)^{-2}
$$

So that Eq. (4.8) can be written

$$
(2+\delta)(1+\delta)^{4}<T / \tau_{0} .
$$

Therefore we see that it is compatible to take $T \gg \tau_{0}$ and $\delta$ of order unity, which means relativistic velocities. In fact, $\delta \sim 1$ corresponds to $v^{2} \sim 0.75 c^{2}$. We must see whether or not this value for $T$ is reasonable. With the considerations made above, we can relate the period with the radius of the orbit. In fact, for a circular orbit

$$
v=\frac{2 \pi r}{T}
$$

and, if we take

$$
\delta \sim 1 \Longrightarrow v^{2} / c^{2} \sim \frac{3}{4},
$$

whence it follows

$$
T^{2} \sim \frac{16}{3} \frac{\pi^{2} r^{2}}{c^{2}}
$$

As we have said above, the radius of the orbit must be much bigger than the classical radius of the electron. Consequently, we have

$$
T \gg \frac{2 \pi r_{c}}{c} \sim 6 \times 10^{-23} \mathrm{sec} .
$$

As $\tau_{0} \sim 6 \times 10^{-24} \mathrm{sec}$, the condition $T \gg \tau_{0}$ is compatible with (4.10).

Let us see in more detail why the nonexistence of a correlation time poses no problem. The condition $\tau_{c} \ll<\tau_{r}^{\text {st }}$ is necessary in order that the cumulant expansion appearing in the theory of the Markov approximation is convergent, at least asymptotically. If there is not a correlation time, we need another parameter in order to guarantee the convergence.

In order to see what this parameter is, let us note that our calculations are made by introducing a cutoff in the spectrum, which is written $\omega^{2} \exp (-\epsilon \omega)$ instead of $\omega^{2}$. After obtaining the Fokker-Planck equation with that spectrum it is seen that everything in this equation is finite in the limit $\epsilon \rightarrow 0$. Inspection of the expression for the diffusion coefficient shows that the convergence depends on the fact that the contribution of the high frequencies to the deterministic motion decreases quickly enough. In other words, the deterministic motion produces an effective cutoff and we have an effective correlation time of the order of the deterministic period. This allows us to work with a spectrum of $\omega^{2}$ type which has not a definite correlation time.

Before concluding, a few words are convenient to clarify the real meaning of the results obtained. Let us consider a material system in a cavity with walls which reflect perfectly at all frequencies. We assume that at the initial time there is no radiation in the cavity and the material system has a finite energy. Then the system radiates and, after some time, the spectrum of the radiation should approach the Rayleigh-Jeans spectrum at low frequencies. The exchange of energy between the system and the radia- tion at high frequencies is slower due to the fact that a Fourier analysis of a deterministic orbit gives a small contribution to high harmonics. Therefore, we arrive at a nonstationary state such that the spectrum of the radiation is of the form $\omega^{2} \Omega(\omega)$, where $\Omega(\omega)$ is a function which decreases at high $\omega$ more quickly than $\omega^{-3}$. The function changes slightly with time so that it decreases at low frequencies and increases at high frequencies.

To see this clearly, we point out that, considering the spectrum with the cutoff, as has in fact been worked out in this paper, for $\epsilon$ small enough, the equilibrium solution is still Maxwell-Boltzmann, and also the radiative equilibrium still holds when one neglects terms of order $\epsilon$. Then, the exchange of energy between the system and the radiation will be slower and slower and the equilibrium is never attained. Obviously, this type of problem is not specific of a relativistic theory and it was extensively discussed at the beginning of the century giving rise to the birth of quantum theory.

\section{APPENDIX A}

The equations of motion for the system that we consider can be written

$$
\dot{\zeta}_{i}=F_{i}(\zeta)+\alpha^{2} F_{i}^{d}+\alpha K_{i}, \quad i=1,2, \ldots, 6
$$

with

$$
\zeta_{i}=x_{i} \text { and } \zeta_{i+3}=p_{i}, \quad i=1,2,3
$$

and where $F_{i}$ is the deterministic force, $\alpha^{2} F_{i}^{d}$ the radiation reaction, and $\alpha K_{i}$ the background electromagnetic field. The constant $\alpha$, which is of order $e$, gives a measure of the intensity of the radiation reaction and the background field. We shall assume that the effect of these forces is small in a deterministic period and besides, that the correlation of the stochastic field decreases quickly with a correlation time $\tau_{c}$ such that $\alpha \tau_{c} \ll<$, which corresponds to conditions (4.2a) and (4.2b). These are the conditions for the validity of the reduced Fokker-Planck equation used in Ref. 1 for the calculation of the probability distribution in the equilibrium state.

Our aim is the calculation of the correlation

$$
\left\langle\overrightarrow{\mathrm{E}}_{\omega, \Delta \omega}(\zeta, t) \rho(\zeta, t)\right\rangle,
$$

$\overrightarrow{\mathbf{E}}_{\omega, \Delta \omega}$ being the component of the electric field with frequencies between $\omega$ and $\omega+\Delta \omega$, and $\rho$ the probability distribution in phase space. To do that we write the continuity equation for $\rho$,

$$
\frac{\partial \rho}{\partial t}=\mathscr{L}_{\alpha} \rho+\mathscr{L}_{1} \rho
$$

where $\mathscr{L}_{0}$ represents the unperturbed motion

$$
\mathscr{L}_{\alpha} \rho=-\sum_{i=1}^{6} \frac{\partial}{\partial \xi_{i}}\left(F_{i} \rho\right)
$$

and $\mathscr{L}_{1}$ the perturbation 


$$
\mathscr{L}_{1} \rho=-\sum_{i=1}^{6} \frac{\partial}{\partial \zeta_{i}}\left[\rho\left(\alpha^{2} F_{i}^{d}+\alpha K_{i}\right)\right]
$$

We introduce the interaction picture, defining

$$
\widetilde{\rho}=e^{-\mathscr{L}_{0} t} \rho,
$$

so that

$$
\begin{aligned}
\frac{\partial}{\partial t} \widetilde{\rho}(\zeta, t) & =e^{-\mathscr{L}_{0} t} \mathscr{L}_{1}(t) e^{\mathscr{L}_{0} t} \widetilde{\rho} \\
& =\widetilde{\mathscr{L}}_{1}(t) \widetilde{\rho}(\zeta, t) .
\end{aligned}
$$

The solution can be written in the usual form

$$
\widetilde{\rho}(\xi, t)=\stackrel{\leftarrow}{\mathrm{T}}\left[\exp \left[\int_{0}^{t} d t^{\prime} \widetilde{\mathscr{L}}_{1}\left(t^{\prime}\right)\right]\right] \widetilde{\rho}_{0}
$$

where $\overleftarrow{T}$ is the operator of time ordering, i.e.,

$$
\overleftarrow{\mathrm{T}}\left[\exp \left[\int_{0}^{t} d t^{\prime} \widetilde{\mathscr{L}}_{1}\left(t^{\prime}\right)\right]\right]=\sum_{n=0}^{\infty} \int_{0}^{t} d t_{1} \int_{0}^{t_{1}} d t_{2} \cdots \int_{0}^{t_{n-1}} d t_{n} \widetilde{\mathscr{L}}_{1}\left(t_{1}\right) \widetilde{\mathscr{L}}_{1}\left(t_{2}\right) \cdots \widetilde{\mathscr{L}}_{1}\left(t_{n}\right)
$$

and $\widetilde{\rho}_{0}=\rho_{0}$. Then

$$
\rho(\xi, t)=e^{\mathscr{L}_{0} t} \overleftarrow{\mathrm{T}}\left[\exp \left[\int_{0}^{t} d t^{\prime} \tilde{\mathscr{L}}_{1}\left(t^{\prime}\right)\right]\right] \rho_{0}
$$

If we average over the samples of the stochastic process and assume that $\rho_{0}$ is uncorrelated with the process, we have

$$
\langle\rho(\zeta, t)\rangle=e^{\mathscr{L}_{0} t}\left\langle\overleftarrow{\mathrm{T}}\left[\exp \left[\int_{0}^{t} d t^{\prime} \widetilde{\mathscr{L}}_{1}\left(t^{\prime}\right)\right]\right]\right\rangle \rho_{0}
$$

From this result and Eq. (A9) we obtain

$$
\rho(\xi, t)=e^{\mathscr{L}_{0} t} \overleftarrow{\mathrm{T}}\left[\exp \left[\int_{0}^{t} d t^{\prime} \mathscr{L}_{1}\left(t^{\prime}\right)\right]\right]\left\langle\overleftarrow{\mathrm{T}}\left[\exp \left[\int_{0}^{t} d t^{\prime} \widetilde{\mathscr{L}}_{1}\left(t^{\prime}\right)\right]\right]\right\rangle^{-1} e^{-\mathscr{L}_{0} t}\langle\rho(\zeta, t)\rangle
$$

Finally, the desired correlation is

$$
\left\langle e \overrightarrow{\mathrm{E}}_{\omega, \Delta \omega}(\zeta, t) \rho(\zeta, t)\right\rangle=\left\langle e \overrightarrow{\mathrm{E}}_{\omega, \Delta \omega}(\zeta, t) e^{\mathscr{L}_{0} t \overleftarrow{\mathrm{T}}}\left[\exp \left[\int_{0}^{t} d t^{\prime} \widetilde{\mathscr{L}}_{1}\left(t^{\prime}\right)\right]\right]\right\rangle\left\langle\overleftarrow{\mathrm{T}}\left[\exp \left[\int_{0}^{t} d t^{\prime} \widetilde{\mathscr{L}}_{1}\left(t^{\prime}\right)\right]\right]\right\rangle^{-1} e^{-\mathscr{L}_{0} t}\langle\rho(\zeta, t)\rangle
$$

that we shall write as

$$
\left\langle e \overrightarrow{\mathrm{E}}_{\omega, \Delta \omega}(\zeta, t) \rho(\zeta, t)\right\rangle=\overrightarrow{\mathrm{U}}(\omega, \Delta \omega, t, \zeta)\langle\rho(\zeta, t)\rangle .
$$

In the limit $t \rightarrow \infty$, we know that $\langle\rho(\zeta, t)\rangle \rightarrow W_{0}$, so that we must calculate the operator $\overrightarrow{\mathrm{U}}(\omega, \Delta \omega, t, \zeta)$. To do that we define the operator

$$
\overrightarrow{\widetilde{\mathrm{U}}}\left(\omega, \Delta \omega, t, t^{\prime}, \zeta\right)=\left\langle e \overrightarrow{\mathrm{E}}_{\omega, \Delta \omega}\left(\zeta, t^{\prime}\right) e^{\mathscr{L}_{0} t \overleftarrow{\mathrm{T}}}\left[\exp \left[\int_{0}^{t} d t^{\prime \prime} \widetilde{\mathscr{L}}_{1}\left(t^{\prime \prime}\right)\right]\right]\right\rangle\left\langle\overleftarrow{\mathrm{T}}\left[\exp \left[\int_{0}^{t} d t^{\prime \prime} \widetilde{\mathscr{L}}_{1}\left(t^{\prime \prime}\right)\right]\right]\right\rangle^{-1},
$$

so that

$$
\overrightarrow{\mathrm{U}}(\omega, \Delta \omega, t, \zeta)=\overrightarrow{\widetilde{U}}(\omega, \Delta \omega, t, t, \zeta)
$$

Now, taking into account (3.1) we see that $\overrightarrow{\mathrm{E}}_{\omega, \Delta \omega}$ can be written as

$$
\overrightarrow{\mathrm{E}}_{\omega, \Delta \omega}(\zeta, t)=\operatorname{Re} \int_{\omega}^{\omega+\Delta \omega} d \omega^{\prime} e^{-i \omega^{\prime} t} \vec{\Phi}\left(\omega^{\prime}, \zeta\right)
$$

where $\vec{\Phi}$ is a process which can be obtained trivially from (3.1). Therefore $\overrightarrow{\widetilde{U}}$ can be written

$$
\overrightarrow{\widetilde{U}}\left(\omega, \Delta \omega, t, t^{\prime}, \zeta\right)=\operatorname{Re} \int_{\omega}^{\omega+\Delta \omega} d \omega^{\prime} e^{-i \omega^{\prime} t^{\prime}} \overrightarrow{\mathscr{U}}\left(\omega^{\prime}, t, \zeta\right),
$$

where $\overrightarrow{\mathscr{U}}$ is given by the expression obtained putting $\vec{\Phi}$ instead of $\vec{E}_{\omega, \Delta \omega}$ in (A14).

Taking into account that

$$
\sum_{n=0}^{\infty} \overrightarrow{\mathrm{E}}_{n \Delta \omega, \Delta \omega}(\xi, t)=\overrightarrow{\mathrm{E}}(\zeta, t),
$$

we obtain, from Eqs. (A17) and (A14),

$$
\begin{aligned}
& \overrightarrow{\mathrm{B}}\left(t, t^{\prime}, \zeta\right)=\sum_{n=0}^{\infty} \overrightarrow{\widetilde{\mathrm{U}}}\left(n \Delta \omega, \Delta \omega, t, t^{\prime}, \zeta\right) \\
& =\operatorname{Re} \int_{0}^{\infty} d \omega e^{-i \omega t^{\prime}} \overrightarrow{\mathscr{U}}(\omega, t, \zeta) \\
& =\left\langle e \overrightarrow{\mathrm{E}}\left(\zeta, t^{\prime}\right) e^{\mathscr{L}_{0} t} \overleftarrow{\mathrm{T}}\left[\exp \left[\int_{0}^{t} d t^{\prime} \widetilde{\mathscr{L}}_{1}\left(t^{\prime}\right)\right]\right]\right\rangle\left\langle\overleftarrow{\mathrm{T}}\left[\exp \left[\int_{0}^{t} d t^{\prime} \widetilde{\mathscr{L}}_{1}\left(t^{\prime}\right)\right]\right]\right\rangle^{-1}
\end{aligned}
$$


where the correlation involves now the background field with all frequencies. From $\overrightarrow{\mathbf{B}}\left(t, t^{\prime}, \zeta\right)$ it is trivial to obtain $\overrightarrow{\mathscr{U}}(\omega, t, \xi)$ in the form

$$
\overrightarrow{\mathscr{U}}(\omega, t, \zeta)=\frac{1}{\pi} \int_{-\infty}^{+\infty} \overrightarrow{\mathrm{B}}\left(t, t^{\prime}, \zeta\right) e^{i \omega t^{\prime}} d t^{\prime} \quad(\omega>0)
$$

Now we study a series expansion for $\overrightarrow{\mathrm{B}}\left(t, t^{\prime}, \zeta\right)$ and we will analyze the behavior of the resulting terms, as regards their order of magnitude when their Fourier transform is made in order to calculate $\overrightarrow{\mathscr{U}}(\omega, t, \zeta)$.

The expansion can be obtained from (A19) by means of a technique, due to Van Kampen, ${ }^{15}$ which gives rise to a cumulant series. Note that in Ref. 15 , instead of $\overrightarrow{\mathrm{E}}\left(\xi, t^{\prime}\right) e^{\mathscr{L}_{0} t}$, there appears the same operator $\mathscr{L}_{1}$ at time $t$; nevertheless, this fact does not influence the formal expansion. The result which we obtain can be written in the form

$$
\overrightarrow{\mathbf{B}}\left(t, t^{\prime}, \zeta\right)=\sum_{m=1}^{\infty} \overrightarrow{\mathrm{B}}_{m}\left(t, t^{\prime}, \zeta\right)
$$

where

$$
\left.\overrightarrow{\mathrm{B}}_{m}\left(t, t^{\prime}, \zeta\right)=\int_{0}^{t} d t_{1} \int_{0}^{t_{1}} d t_{2} \cdots \int_{0}^{t_{m-2}} d t_{m-1}\left\langle e \overrightarrow{\mathrm{E}}\left(\xi, t^{\prime}\right) e^{\mathscr{L}_{0} t} \widetilde{\mathscr{L}}_{1}\left(t_{1}\right) \cdots \widetilde{\mathscr{L}}_{1}\left(t_{m-1}\right)\right\rangle\right\rangle
$$

and $\left\langle\langle\cdots\rangle\right.$ represents the ordered cumulants of Van Kampen. ${ }^{15}$

The cumulants have the "cluster" property which means that they are negligible if two contiguous times differ more than the correlation time of the background field. This is the reason we have introduced an expansion for $\overrightarrow{\mathbf{B}}\left(t, t^{\prime}, \zeta\right)$ and not directly for $\overrightarrow{\widetilde{U}}\left(\omega, \Delta \omega, t, t^{\prime}, \xi\right)$, because in that case the first component should be $\overrightarrow{\mathrm{E}}_{\omega, \Delta \omega}$ whose correlation with the background field is oscillatory and does not decrease with time. However, for the total field $\overrightarrow{\mathrm{E}}$ the correlation decreases in a time interval of order $\tau_{c}$ and the desired property of the cumulants is fulfilled. As a consequence of this property, each term $\overrightarrow{\mathrm{B}}_{m}$ is of order $\alpha\left(\alpha \tau_{c}\right)^{m-1}$ and the expansion (A21) converges asymptotically, while a similar expansion for $\widetilde{U}$ requires a more careful analysis, which we make in the following.

The Fourier transform of (A21) gives

$$
\overrightarrow{\mathscr{U}}(\omega, t, \zeta)=\sum_{m=1}^{\infty} \overrightarrow{\mathscr{U}}_{m}(\omega, t, \zeta)
$$

with

$$
\overrightarrow{\mathscr{U}}_{m}(\omega, t, \zeta)=\frac{1}{\pi} \int_{-\infty}^{+\infty} \overrightarrow{\mathrm{B}}_{m}\left(t, t^{\prime}, \zeta\right) e^{i \omega t^{\prime}} d t^{\prime} .
$$

Now, because of the cluster property, in Eq. (A22) $\left|t^{\prime}-t\right| \leqslant \tau_{c}$, and as $t_{1}$ is integrated between 0 and $t$, it is clear that $\overrightarrow{\mathrm{B}}_{m}$ contributes only if $t^{\prime}$ belongs to the interval $\left(-\tau_{c}, t+\tau_{c}\right)$, which, for $t \gg \tau_{c}$, coincides practically with $(0, t)$. After that, it is trivial that each $\overrightarrow{\mathscr{U}}_{m}$ is bound by a quantity of order $t \alpha\left(\alpha \tau_{c}\right)^{m-1}$. As we are interested in the stationary state, $t \rightarrow \infty$ and that order-of-magnitude estimation loses in principle its meaning. However, we shall see that the expansion is yet valid. To do that, we write the contribution to the $m$ th term to the correlation (A12),

$$
\begin{aligned}
\overrightarrow{\mathbf{C}}_{m}(t)=\operatorname{Re} \int_{\omega}^{\omega+\Delta \omega} d \omega^{\prime} e^{-i \omega^{\prime} t} \frac{1}{\pi} \int_{-\infty}^{+\infty} e^{i \omega^{\prime} t^{\prime}} d t^{\prime} & \\
\times \int_{0}^{t} d t_{1} \int_{0}^{t_{1}} d t_{2} \cdots \int_{0}^{t_{m-2}} d t_{m-1} & \left\langle e \overrightarrow{\mathrm{E}}\left(\xi, t^{\prime}\right) e^{\mathscr{L}_{0}\left(t-t_{1}\right)} \mathscr{L}_{1}\left(\xi, t_{1}\right)\right. \\
& \times e^{\mathscr{L}_{0}\left(t_{1}-t_{2}\right)} \mathscr{L}_{1}\left(\zeta, t_{2}\right) \cdots e^{\mathscr{L}_{0}\left(t_{m-2}-t_{m-1}\right)} \\
& \left.\times \mathscr{L}_{1}\left(\xi, t_{m-1}\right)\right\rangle e^{\mathscr{L}_{0}\left(t_{m-1}-t\right)}\langle\rho(\zeta, t)\rangle,
\end{aligned}
$$

so that

$$
\left\langle e \overrightarrow{\mathrm{E}}_{\omega, \Delta \omega}(\zeta, t) \rho(\zeta, t)\right\rangle=\sum_{m=1}^{\infty} \overrightarrow{\mathrm{C}}_{m}(t),
$$

and let us make the changes of variable

$$
\begin{aligned}
& t-t_{1}=u, \\
& t^{\prime}-t_{1}=\tau_{1}, \\
& t_{1}-t_{2}=\tau_{2}, \\
& \ldots \\
& t_{m-2}-t_{m-1}=\tau_{m-1}
\end{aligned}
$$


Then Eq. (A25) is written

$$
\begin{gathered}
\overrightarrow{\mathrm{C}}_{m}(t)=\operatorname{Re} \int_{\omega}^{\omega+\Delta \omega} d \omega^{\prime} \frac{1}{\pi} \int_{-\infty}^{+\infty} d \tau_{1} e^{i \omega^{\prime} \tau_{1}} \int_{0}^{t} d u e^{-i \omega^{\prime} u} \int_{0}^{t-u} d \tau_{2} \int_{0}^{t-u-\tau_{2}} d \tau_{3} \cdots \\
\times \int_{0}^{t-u-\left(\tau_{2}+\cdots+\tau_{m-2}\right)} d \tau_{m-1}\left\langle\left\langle e \overrightarrow{\mathrm{E}}\left(\zeta, \tau_{1}\right) e^{\mathscr{L}_{0} u} \mathscr{L}_{1}(\zeta, 0) e^{\mathscr{L}_{0} \tau_{2}} \mathscr{L}_{1}\left(\zeta,-\tau_{2}\right)\right.\right. \\
\times e^{\mathscr{L}_{0} \tau_{3}} \mathscr{L}_{1}\left(\xi,-\tau_{2}-\tau_{3}\right) \cdots e^{\left.\left.\mathscr{L}_{0} \tau_{m-1} \mathscr{L}_{1}\left(\zeta,-\left[\tau_{2}+\cdots+\tau_{m-1}\right]\right)\right\rangle\right\rangle} \\
\times e^{\mathscr{L}_{0}\left(-u-\tau_{2}-\cdots-\tau_{m-1}\right)}\langle\rho(\zeta, t)\rangle,
\end{gathered}
$$

where we have subtracted the time $t-u$ in every $\mathscr{L}_{1}$, which is possible for a stationary process.

After that, for the cluster property of the cumulants, these are negligible when the time interval between two successive operators $\mathscr{L}_{1}$ is larger than the correlation time. Moreover, the deterministic motion does not give rise to divergences, the motion being multiply periodic. Then $\tau_{i} \lesssim \tau_{c}$ and the integrals over $\tau_{i}$ give rise to a term of order $\left(\alpha \tau_{c}\right)^{m-1}$. Therefore the possible divergences appear only in the $u$ integral, which we study in the following.

Let us divide the interval of that integral in two parts,

$$
\int_{0}^{t} d u \cdots=\int_{0}^{t-(m-2) \tau_{c}} d u \cdots+\int_{t-(m-2) \tau_{c}}^{t} d u \cdots
$$

The second contribution presents an integration interval $(m-2) \tau_{c}$ so that this part is of order $(m-2) \tau_{c}\left(\alpha \tau_{c}\right)^{m-1}$ even in the limit $t \rightarrow \infty$. With respect to the first part, we note that the conditions

$$
\begin{aligned}
& 0 \leq \tau_{2} \leq \tau_{c}, \\
& 0 \leq \tau_{3} \leq \tau_{c}, \\
& \cdots \\
& 0 \leq \tau_{m-1} \leq \tau_{c}, \\
& 0 \leq u \leq t-(m-2) \tau_{c}
\end{aligned}
$$

imply

$$
\begin{aligned}
& \tau_{2} \leq t-u, \\
& \tau_{3} \leq t-u-\tau_{2}, \\
& \cdots \\
& \tau_{m-1} \leq t-u-\left(\tau_{2}+\cdots+\tau_{m-2}\right),
\end{aligned}
$$

whence it follows that

$$
\begin{aligned}
& {\left[0, t-(m-2) \tau_{c}\right] \times \overbrace{\left[0, \tau_{c}\right] \times\left[0, \tau_{c}\right] \times \cdots \times\left[0, \tau_{c}\right]}^{m-2}} \\
& \quad \subset\left[0, t-(m-2) \tau_{c}\right] \times[0, t-u] \times\left[0, t-u-\tau_{2}\right] \times \cdots \times\left[0, t-u-\left(\tau_{2}+\cdots+\tau_{m-2}\right)\right]
\end{aligned}
$$

and again the cluster property of the cumulants leads to the result that the contribution of the first term of the righthand side of (A29) to (A28) is of the order of

$$
\begin{aligned}
& \overrightarrow{\mathrm{C}}_{m}^{(1)}(t) \cong \operatorname{Re} \int_{\omega}^{\omega+\Delta \omega} d \omega^{\prime} \frac{1}{\pi} \int_{-\tau_{c}}^{\tau_{c}} d \tau_{1} e^{i \omega^{\prime} \tau_{2}} \int_{0}^{t-(m-2) \tau_{c}} d u e^{-i \omega^{\prime} u} \\
& \times \int_{0}^{\tau_{c}} d \tau_{2} \int_{0}^{\tau_{c}} d \tau_{3} \cdots \int_{0}^{\tau_{c}} d \tau_{m-1}\left\langle e \mathrm{E}\left(\zeta, \tau_{1}\right) e^{\mathscr{L}_{0} u} \mathscr{L}_{1}(\zeta, 0) e^{\mathscr{L}_{0} \tau_{2}} \mathscr{L}_{1}\left(\zeta,-\tau_{2}\right) \cdots\right. \\
&\left.\times e^{\mathscr{L}_{0} \tau_{m-1}} \mathscr{L}_{1}\left(\zeta,-\left[\tau_{2}+\cdots+\tau_{m-2}\right]\right)\right\rangle \\
& \times e^{\mathscr{L}_{0}\left(-u-\tau_{2}-\cdots-\tau_{m-1}\right)}\langle\rho(\zeta, t)\rangle,
\end{aligned}
$$

and, assuming that $t$ is large enough, 
$\overrightarrow{\mathrm{C}}_{m}^{(1)}(t) \underset{t \rightarrow \infty}{\simeq} \operatorname{Re} \int_{\omega}^{\omega+\Delta \omega} d \omega^{\prime} \frac{1}{\pi} \int_{-\tau_{c}}^{\tau_{c}} d \tau_{1} e^{i \omega^{\prime} \tau_{1}} \int_{0}^{t} d u e^{-i \omega^{\prime} u} \int_{0}^{\tau_{c}} d \tau_{2}$

$$
\begin{aligned}
\times \int_{0}^{\tau_{c}} d \tau_{2} \int_{0}^{\tau_{c}} d \tau_{3} \cdots \int_{0}^{\tau_{c}} d \tau_{m-1}\langle & e \overrightarrow{\mathrm{E}}\left(\zeta, \tau_{1}\right) e^{\mathscr{L}_{0} u} \mathscr{L}_{1}(\zeta, 0) e^{\mathscr{L}_{0} \tau_{2}} \mathscr{L}_{1}\left(\zeta,-\tau_{2}\right) \cdots \\
& \left.\times e^{\mathscr{L}_{c} \tau_{m-1}} \mathscr{L}_{1}\left(\zeta,-\left[\tau_{2}+\cdots+\tau_{m-1}\right]\right)\right\rangle W_{0}(\zeta),
\end{aligned}
$$

where we have used

$$
\langle\rho(\zeta, t)\rangle \underset{t \rightarrow \infty}{\rightarrow} W_{0}[\mathscr{E}(\overrightarrow{\mathrm{J}})]
$$

and we have taken into account that $\mathscr{E}$ is a constant with respect to the deterministic motion.

Now we can analyze the divergences that appear in (A33) when we take the limit $t \rightarrow \infty$. We see that $u$ appears in (A33) due to the action of the classical Liouvillian on the phase-space coordinates. Therefore, we will have, on the right of $e^{\mathscr{L}_{0} u}$, some function

$$
\varphi\left(\zeta, \tau_{2}, \ldots, \tau_{m-1}\right)
$$

and, due to the properties of the action-angle variables,

$$
\begin{aligned}
& \varphi\left(\xi, \tau_{2}, \ldots, \tau_{m-1}\right) \\
& =\sum_{\overrightarrow{\mathrm{n}}} \varphi_{\overrightarrow{\mathrm{n}}}\left(\overrightarrow{\mathbf{J}}, \overrightarrow{\mathbf{J}}^{\prime}, \overrightarrow{\mathrm{w}}^{\prime}, \tau_{2}, \ldots, \tau_{m-1}\right) e^{-i \overrightarrow{\mathrm{n}} \cdot \overrightarrow{\mathrm{w}}},
\end{aligned}
$$

so that

$e^{\mathscr{L}_{0} u} \varphi=\sum_{\overrightarrow{\mathrm{n}}} \varphi_{\overrightarrow{\mathrm{n}}}\left(\overrightarrow{\mathrm{J}}, \overrightarrow{\mathrm{J}}^{\prime}, \overrightarrow{\mathrm{w}}^{\prime}, \tau_{2}, \ldots, \tau_{m-1}\right) e^{-i \overrightarrow{\mathrm{n}} \cdot \overrightarrow{\mathrm{w}}} e^{i \overrightarrow{\mathrm{n}} \cdot \vec{\omega}_{0} u}$
Therefore, the integral on $u$ gives rise to a term of the form

$$
\int_{0}^{t} d u e^{-i \omega u} e^{i \overrightarrow{\mathrm{n}} \cdot \vec{\omega}_{0}^{u}}=\frac{e^{-i u\left(\omega-\overrightarrow{\mathrm{n}} \cdot \vec{\omega}_{0}\right)}-1}{-i\left(\omega-\overrightarrow{\mathrm{n}} \cdot \vec{\omega}_{0}\right)},
$$

which is bounded if $\omega \neq \overrightarrow{\mathrm{n}} \cdot \vec{\omega}_{0}$ and increases as $t$ for $\omega=\overrightarrow{\mathbf{n}} \cdot \vec{\omega}_{0}$.

As a consequence, the divergences appear at those frequencies $\omega$ which agree with some frequency of the multiply periodic motion. The important thing is that in each term of the cumulant expansion all frequencies of the multiply periodic motion appear. Therefore, every one behaves as $t$ if $\omega$ coincides with some frequency $\vec{n} \cdot \vec{\omega}_{0}$ and are bound in the opposite case. As the remaining contributions to $C_{m}^{(1)}$ are of order $\left(\alpha \tau_{c}\right)^{m-1}$ we obtain, finally, that for frequencies $\omega \neq \overrightarrow{\mathbf{n}} \cdot \vec{\omega}_{0} \forall \overrightarrow{\mathbf{n}}, C_{m} \sim \tau_{c}\left(\alpha \tau_{c}\right)^{m-1}$ and if $\omega=\overrightarrow{\mathrm{n}} \cdot \vec{\omega}_{0}$ for some $\overrightarrow{\mathrm{n}}, C_{m} \sim t\left(\alpha \tau_{c}\right)^{m-1}$ for all $t$.

As a consequence, the expansion found is valid and we can take the first nonzero term only, namely $C_{2}$ because $C_{1} \equiv 0$, in the estimate of the correlation (A12),

$\left\langle e \overrightarrow{\mathrm{E}}_{\omega, \Delta \omega}(\xi, t) \rho(\xi, t)\right\rangle \cong \operatorname{Re} \int_{\omega}^{\omega+\Delta \omega} d \omega^{\prime} \frac{1}{\pi} \int_{-\infty}^{+\infty} d \tau_{1} e^{i \omega^{\prime} \tau_{1}} \int_{0}^{t} d u e^{-i \omega^{\prime} u}\left\langle\left\langle e \overrightarrow{\mathrm{E}}\left(\zeta, \tau_{1}\right) e^{\mathscr{L}_{0} u} \mathscr{L}_{1}(\xi, 0)\right\rangle e^{-\mathscr{L}_{0} u}\langle\rho(\zeta, t)\rangle\right.$

As the second-order cumulant is the correlation and the mean value of the background field is zero, in Eq. (A35) only the fluctuating part of $\mathscr{L}_{1}$ contributes,

$$
\mathscr{L}_{1}-\left\langle\mathscr{L}_{1}\right\rangle=-\sum_{i} \frac{\partial}{\partial \xi_{i}}\left(\alpha K_{i} \cdot\right)
$$

In our case, the stochastic force has only components $4,5,6, \alpha K_{i+3}=F_{i}^{\text {st }}$ and, besides, ${ }^{1}$

$$
\sum_{i=1}^{6} \frac{\partial}{\partial \xi_{i}}\left(\alpha K_{i}\right)=\sum_{i=1}^{3} \frac{\partial}{\partial p_{i}}\left(F_{i}^{\mathrm{st}}\right)=0
$$

so that

$$
\mathscr{L}_{1}-\left\langle\mathscr{L}_{1}\right\rangle=-\sum_{i=1}^{3} F_{i}^{\mathrm{st}}(\overrightarrow{\mathrm{r}}, \overrightarrow{\mathrm{p}}, t) \frac{\partial}{\partial p_{i}} .
$$

On the other hand, the evolution operator of the multiply periodic system verifies ${ }^{12}$

$$
e^{\mathscr{L}_{0} u} \mathscr{L}_{1}(\zeta, 0) e^{-\mathscr{L}_{0} u}\langle\rho(\zeta, t)\rangle=\mathscr{L}_{1}\left(\xi^{-u}, 0\right)\langle\rho(\zeta, t)\rangle .
$$

After that, the expression (A35) can be written

$$
\begin{aligned}
\left\langle e \overrightarrow{\mathrm{E}}_{\omega, \Delta \omega}(\zeta, t) \rho(\zeta, t)\right\rangle \simeq-\int_{\omega}^{\omega+\Delta \omega} d \omega^{\prime} \frac{1}{\pi} \int_{-\infty}^{+\infty} d \tau_{1} \int_{0}^{t} d u \cos \omega^{\prime}\left(\tau_{1}-u\right) & \\
& \times\left\langle\left\langle e \overrightarrow{\mathrm{E}}\left(\overrightarrow{\mathrm{r}}, \tau_{1}\right) \sum_{i=1}^{3} F_{i}^{\mathrm{st}}\left(\overrightarrow{\mathrm{r}}^{-u}, \overrightarrow{\mathrm{p}}^{-u}, 0\right)\right\rangle \frac{\partial}{\partial p_{i}^{-u}}\langle\rho(\zeta, t)\rangle\right.
\end{aligned}
$$


The correlation of the background field can be written in terms of the expressions of Ref. 1,

$$
\left\langle E_{i}\left(\overrightarrow{\mathrm{r}}, \tau_{1}\right) F_{j}^{\mathrm{st}}\left(\overrightarrow{\mathrm{r}}^{-u}, \overrightarrow{\mathrm{p}}^{-u}, 0\right)\right\rangle=e \int_{0}^{\infty} d \omega \frac{\omega^{2} \mathscr{Y}^{2}(\omega)}{2 c^{3}} \int d \Omega T_{i j}\left(\overrightarrow{\mathrm{V}}^{-u}\right) \cos \left[\omega \frac{\hat{r}^{0}}{c} \cdot\left(\overrightarrow{\mathrm{r}}-\overrightarrow{\mathrm{r}}^{-u}\right)-\omega \tau_{1}\right]
$$

with

$$
T_{i j}(\overrightarrow{\mathrm{V}})=\left(\delta_{i j}-r_{i}^{0} r_{j}^{0}\right)+\sum_{k r l} \epsilon_{j k r} \epsilon_{i r l} r_{l}^{0} V_{k}
$$

Substituting (A39) into (A38) and integrating over $\tau_{1}$, we obtain

$$
\begin{aligned}
&\left\langle e\left(E_{\omega, \Delta \omega}(\zeta, t)\right)_{i} \rho(\zeta, t)\right\rangle \cong-\int_{\omega}^{\omega+\Delta \omega} d \omega^{\prime} e^{2} \int_{0}^{t} d u \int_{0}^{\infty} d \omega^{\prime \prime} \frac{\omega^{\prime \prime 2} \mathscr{Y}^{2}\left(\omega^{\prime \prime}\right)}{2 c^{3}} \\
& \quad \times \sum_{j=1}^{3} \int d \Omega T_{i j}\left(\overrightarrow{\mathrm{V}}^{-u}\right) \delta\left(\omega^{\prime}-\omega^{\prime \prime}\right) \cos \left[\omega^{\prime} \frac{\hat{r}^{0}}{c} \cdot\left(\overrightarrow{\mathrm{r}}-\overrightarrow{\mathrm{r}}^{-u}\right)-\omega^{\prime} u\right] \frac{\partial}{\partial p_{j}^{-u}}\langle\rho(\zeta, t)\rangle \\
&=-\int_{\omega}^{\omega+\Delta \omega} d \omega^{\prime} \frac{e^{2} \omega^{\prime 2} \mathscr{Y}^{2}\left(\omega^{\prime}\right)}{2 c^{3}} \\
& \quad \times \int_{0}^{t} d u \int d \Omega \sum_{j=1}^{3} T_{i j}\left(\overrightarrow{\mathrm{V}}^{-u}\right) \cos \left[\omega^{\prime} \frac{\hat{r}^{0}}{c} \cdot\left(\overrightarrow{\mathrm{r}}-\overrightarrow{\mathrm{r}}^{-u}\right)-\omega^{\prime} u\right] \frac{\partial}{\partial p_{j}^{-u}}\langle\rho(\zeta, t)\rangle,
\end{aligned}
$$

where we have taken into account that $\omega^{\prime}$ and $\omega^{\prime \prime}$ are both positive.

We have seen that this expression gives the main contribution to the correlation for any time $t$. What happens in the limit $t \rightarrow \infty$ ? We must note that in the absorbed intensity, that expression is integrated over phase space which is equivalent to an integration over all deterministic orbits. As the divergences appear due to resonance between characteristic frequencies of the deterministic motion with components of the stochastic field, an integration over phase space of (A41) destroys the resonance effect and the divergences disappear in the limit $t \rightarrow \infty$. If we take the limit before the integral over phase space, there appear Dirac's $\delta$ 's for each frequency $\omega=\vec{n} \cdot \vec{\omega}_{0}$, giving each a finite contribution to that integral. Therefore, the limit $t \rightarrow \infty$ has a sense as a distribution and we obtain expression (3.5).

We must point out that this result agrees to the first order with the one obtained from Kubo linear response theory ${ }^{10}$ for a system which evolves from the stationary state $W_{0}$ under the action of the deterministic force plus the $\omega$ component of the electric background field. This result can be understood because, in the approximation considered, the damping force gives rise to a negligible effect. Besides, to first order, the effect of the field at frequencies different from $\omega$ does not appear, as is the case in Kubo theory.

The application of this result to the problem that we are considering has the same difficulty that appears in the study of the validity of the Markov approximation for the calculation of the probability distribution of the stationary state. The singular nature of the spectrum implies that there is not a true correlation time and we must either make a qualitative analysis of the magnitude of the random force or, alternatively, to start from a more correct theory where the spectrum is cut off and it is possible to define a true correlation time. A more detailed discussion of this point appears in Sec. IV of this paper.

\section{APPENDIX B}

In this appendix we shall study the relation between the emitted and the absorbed power at every frequency, and the Fokker-Planck equation reduced to the constants of the unperturbed motion. This relationship has been studied previously for the nonrelativistic case, ${ }^{6,16}$ where $F^{\text {st }}$ depends only on time. The power emitted at the frequency $\omega, I_{e}(\omega)$, is given by Eq. (2.27). As we have seen in Ref. 1, Sec. VA, the power emitted averaged over an orbit is given by the drift coefficient $G_{1}(\mathscr{E})$ corresponding to a given energy. Therefore, the total power averaged over the whole phase space $I_{e}$ will be given by the average of $G_{1}(\mathscr{E})$ in the reduced space of the constants of motion. To see that from Eq. (2.27) for $I_{e}(\omega)$, it is enough to integrate over frequencies, which leads to

$$
\begin{aligned}
I_{e}=\int_{0}^{\infty} I_{e}(\omega) d \omega \\
=-\frac{e^{2}}{2 \pi c^{2}} \int d \zeta W_{0} \\
\\
\quad \times \sum_{\overrightarrow{\mathrm{n}}}^{\prime}\left(\overrightarrow{\mathrm{n}} \cdot \vec{\omega}_{0}\right)^{2} \operatorname{Re} \sigma_{\overrightarrow{0},-\overrightarrow{\mathrm{n}}}[\mathscr{E}]\left(\overrightarrow{\mathrm{n}} \cdot \vec{\omega}_{0} ; \vec{\omega}_{0}\right)
\end{aligned}
$$

If we take into account that the expression under the in- 
tegral is only a function of the constants of motion

$$
\overrightarrow{\mathbf{J}} \equiv\left(J_{1}, \ldots, J_{M}\right), \quad \overrightarrow{\mathbf{J}}^{\prime} \equiv\left(J_{M+1}, \ldots, J_{3}\right),
$$

and

$$
\overrightarrow{\mathbf{w}}^{\prime} \equiv\left(w_{M+1}, \ldots, w_{3}\right),
$$

we obtain

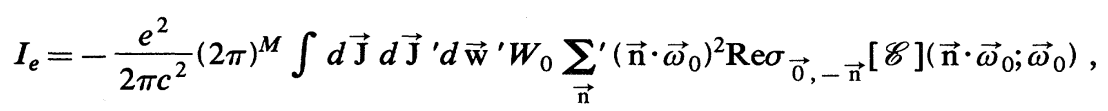

that is [see (5.25) of Ref. 1],

$$
I_{e}=-\int d \overrightarrow{\mathrm{J}} d \overrightarrow{\mathrm{J}}^{\prime} d \overrightarrow{\mathrm{w}}^{\prime} G_{1}(\mathscr{E}) W_{0} .
$$

In order to put (B3) in terms of the drift coefficients appearing in the Fokker-Planck equation [Eq. (4.3) of Ref. 1], that is, $G_{1}(\overrightarrow{\mathrm{J}}), G_{1}\left(\overrightarrow{\mathrm{J}}^{\prime}\right)$, and $G_{1}\left(\overrightarrow{\mathrm{w}}^{\prime}\right)$, we use the relation

$$
G_{1}(\mathscr{C})=\sum_{j=1}^{3} \mathrm{~T}\left[F_{j}^{\mathrm{d}} \frac{\partial \mathscr{C}}{\partial p_{j}}\right]=-\sum_{j=1}^{3} \sum_{i=1}^{M} \frac{\partial \mathscr{E}}{\partial J_{i}} \mathrm{~T}\left[F_{j}^{\mathrm{d}} \frac{\partial J_{i}}{\partial p_{j}}\right]=\sum_{i=1}^{M} \omega_{i} G_{1}\left(J_{i}\right) .
$$

From (B3) and (B4) we obtain

$$
I_{e}=\int_{0}^{\infty} I_{e}(\omega) d \omega=-\int d \overrightarrow{\mathbf{J}} d \overrightarrow{\mathbf{J}}^{\prime} d \overrightarrow{\mathrm{w}}^{\prime} \sum_{i=1}^{M} \omega_{i} G_{1}\left(J_{i}\right) W_{0}
$$

and, with an integration by parts

$$
I_{e}=\int_{0}^{\infty} I_{e}(\omega) d \omega=\int d \overrightarrow{\mathbf{J}} d \overrightarrow{\mathrm{J}}^{\prime} d \overrightarrow{\mathrm{w}}^{\prime} \mathscr{E}\left[\sum_{i=1}^{M} \frac{\partial}{\partial J_{i}}\left[G_{1}\left(J_{i}\right) W_{0}\right]\right],
$$

where the term in large brackets corresponds to the drift part in the Fokker-Planck equation for the variables $\overrightarrow{\mathbf{J}}$. On the other hand, it is obvious that such a term can be replaced in (B6) by the full drift term in the Fokker-Planck equation, in view of the fact that $\mathscr{C}$ depends neither on $\vec{J}^{\prime}$ nor on $\overrightarrow{\mathbf{w}}^{\prime}$. That is, the contribution of the drift part corresponding to $\overrightarrow{\mathrm{J}}$ ' and $\overrightarrow{\mathrm{w}}^{\prime}$ to the power emitted on the average is zero for each frequency. Therefore, we may state, from (B6), that $I_{e}(\omega)$ corresponds to the frequency analysis of $I_{e}$, while the drift term is related to the contribution of each orbit to the emitted power $I_{e}$.

Now, we consider the total absorbed power $I_{a}$, which is given from (3.4), (3.5), and (3.9) by

$$
\begin{aligned}
I_{a} & =\int_{0}^{\infty} I_{a}(\omega) d \omega \\
& =-\frac{e^{2}}{c^{3}} \int_{0}^{\infty} d \omega \int d \zeta \sum_{i, j} V_{i} \mathscr{Y}^{2}(\omega) \omega^{2} \int_{0}^{\infty} d u \int d \Omega T_{i j}\left(\overrightarrow{\mathrm{V}}^{-u}\right) \cos \left(\omega \frac{\hat{r}^{0}}{c} \cdot\left(\overrightarrow{\mathrm{r}}-\overrightarrow{\mathrm{r}}^{-u}\right)-\omega u\right) \frac{\partial}{\partial p_{j}^{-u}} W_{0} .
\end{aligned}
$$

Taking into account (A39) we have

$$
I_{a}=-\int d \zeta \int_{0}^{\infty} d u \sum_{j=1}^{3} \overrightarrow{\mathrm{V}} \cdot\left\langle e \overrightarrow{\mathrm{E}}(\overrightarrow{\mathrm{r}}, 0) \cdot F_{j}^{\mathrm{st}}\left(\overrightarrow{\mathrm{r}}^{-u}, \overrightarrow{\mathrm{p}}^{-u},-u\right)\right\rangle \frac{\partial}{\partial p_{j}^{-u}} W_{0},
$$

where $\overrightarrow{\mathrm{F}}^{\text {st }}=e(\overrightarrow{\mathrm{E}}+\overrightarrow{\mathrm{V}} \times \overrightarrow{\mathrm{B}} / c)$ is the stochastic force due to the radiation field. As $W_{0}$ is a function only of the constants of motion for an arbitrary radiation field and $\vec{V} \cdot \overrightarrow{\mathrm{F}}$ st reduces to $\overrightarrow{\mathrm{V}} \cdot e \overrightarrow{\mathrm{E}}$, we have

$$
I_{a}=-\int d \zeta \int_{0}^{\infty} d u \sum_{i, j=1}^{3} \frac{\partial \mathscr{C}}{\partial p_{i}}\left\langle F_{i}^{\mathrm{st}}(\overrightarrow{\mathrm{r}}, \overrightarrow{\mathrm{p}}, 0) F_{j}^{\mathrm{st}}\left(\overrightarrow{\mathrm{r}}^{-u}, \overrightarrow{\mathrm{p}}^{-u},-u\right)\right\rangle \sum_{v} \frac{\partial W_{0}}{\partial h_{v}} \frac{\partial h_{v}}{\partial p_{j}^{-u}}
$$

where the sum in $v$ indicates all the constants of motion. If we write the integral over phase space as an integral along each orbit, integrated over the constants of motion, we obtain

$$
I_{a}=-\int d \overrightarrow{\mathbf{J}} d \overrightarrow{\mathbf{J}}^{\prime} d \overrightarrow{\mathrm{w}}^{\prime}\left[\sum_{i=1}^{M}\left[\bar{G}_{2}\left(\mathscr{E}, J_{i}\right) \frac{\partial W_{0}}{\partial J_{i}}\right]+\sum_{r=M+1}^{3}\left[\bar{G}_{2}\left(\mathscr{E}, J_{r}^{\prime}\right) \frac{\partial W_{0}}{\partial J_{r}^{\prime}}+\bar{G}_{2}\left(\mathscr{E}, w_{r}^{\prime}\right) \frac{\partial W_{0}}{\partial w_{r}^{\prime}}\right]\right],
$$

where $\bar{G}_{2}\left(\mathscr{C}, h_{v}\right)$ corresponds to the reduced diffusion coefficient [see (4.1b) in Ref. 1].

Writing these coefficients as functions of the ones associated with the action-angle variables from the relation

$$
\bar{G}_{2}\left(\mathscr{E}, h_{v}\right)=\sum_{K=1}^{M} \frac{\partial \mathscr{E}}{\partial J_{K}} \bar{G}_{2}\left(J_{K}, h_{v}\right)=\sum_{K=1}^{M} \omega_{K} \bar{G}_{2}\left(J_{K}, h_{v}\right),
$$


which is obtained in a form similar to (B4), we obtain

$$
I_{a}=-\int d \overrightarrow{\mathbf{J}} d \overrightarrow{\mathbf{J}}^{\prime} d \overrightarrow{\mathrm{w}}^{\prime} \sum_{K=1}^{M} \omega_{K}\left\{\sum_{i=1}^{M} \bar{G}_{2}\left(J_{K}, J_{i}\right) \frac{\partial W_{0}}{\partial J_{i}}+\sum_{r=M+1}^{3}\left[\bar{G}_{2}\left(J_{K}, J_{r}^{\prime}\right) \frac{\partial W_{0}}{\partial J_{r}^{\prime}}+\bar{G}_{2}\left(J_{K}, w_{r}^{\prime}\right) \frac{\partial W_{0}}{\partial w_{r}^{\prime}}\right]\right\}
$$

An integration by parts of this expression finally gives

$$
I_{a}=\int_{0}^{\infty} I_{a}(\omega) d \omega=\int d \overrightarrow{\mathbf{J}} d \overrightarrow{\mathbf{J}}^{\prime} d \overrightarrow{\mathbf{w}}^{\prime} \mathscr{E} \sum_{K=1}^{M} \frac{\partial}{\partial J_{K}}\left\{\sum_{i=1}^{M} \bar{G}_{2}\left(J_{K}, J_{i}\right) \frac{\partial}{\partial J_{i}}+\sum_{r=M+1}^{3}\left[\bar{G}_{2}\left(J_{k}, J_{r}^{\prime}\right) \frac{\partial}{\partial J_{r}^{\prime}}+\bar{G}_{2}\left(J_{K}, w_{r}^{\prime}\right) \frac{\partial}{\partial w_{r}^{\prime}}\right]\right\} W_{0}
$$

Again, as in (B6), we may include on the right-hand side of (B13) the whole diffusion term of the Fokker-Planck equation because $\mathscr{E}$ depends only on $\overrightarrow{\mathrm{J}}$. Consequently, $I_{a}(\omega)$ corresponds to a frequency analysis of $I_{a}$, while the diffusion part of the Fokker-Planck equation is related to the contribution of each orbit to the absorbed power.

If we add (B6) and (B13) including $G_{1}\left(J_{i}^{\prime}\right), G_{1}\left(w_{i}^{\prime}\right), G_{2}\left(J_{i}^{\prime}, h_{v}\right)$, and $\bar{G}_{2}\left(w_{i}^{\prime}, h_{v}\right)$, we have

$$
I_{a}+I_{e}=\int d \overrightarrow{\mathbf{J}} d \overrightarrow{\mathbf{J}}^{\prime} d \overrightarrow{\mathbf{w}}^{\prime} \mathscr{E} \sum_{\mu} \frac{\partial}{\partial h_{\mu}}\left[G_{1}\left(h_{\mu}\right)+\sum_{v} \bar{G}_{2}\left(h_{\mu}, h_{v}\right) \frac{\partial}{\partial h_{v}}\right] W_{0}=0
$$

because $W_{0}$ satisfies the Fokker-Planck equation for the stationary state. This is obvious from a physical point of view because it is necessary to have global energetic equilibrium in the stationary state.

Let us specialize to a relativistic system in a radiation field with Rayleigh-Jeans spectrum. In this case we have shown that $W_{0}$ is given by the Maxwell-Boltzmann distribution ${ }^{1}$ and that there is equilibrium at each frequency, that is, $I_{e}(\omega)+I_{a}(\omega)=0$. We will see that in this case a stronger type of balance is satisfied, which implies not only radiative balance but also detailed balance.

As $W_{0}$ depends only on the energy, $W_{0}[\mathscr{E}(\overrightarrow{\mathrm{J}})]$, we may put (B12) in the form

$$
I_{a}=-\int d \overrightarrow{\mathbf{J}} d \overrightarrow{\mathbf{J}}^{\prime} d \overrightarrow{\mathrm{w}}^{\prime} \sum_{K=1}^{M} \omega_{K} G_{2}^{S}\left(J_{K}\right) \frac{d W_{0}}{d \mathscr{C}},
$$

where we have used the notation of Ref. 1,

$$
G_{2}(\varphi)=\sum_{K=1}^{M} \bar{G}_{2}\left(\varphi, J_{K}\right) \omega_{K}
$$

and the character symmetric of $G_{2}\left(J_{K}\right)$ [see (6.7) in Ref. 1]. Using the expressions (5.25) and $(5.31)$ for $G_{1}(\varphi)$ and $G_{2}^{s}(\varphi)$, respectively given in Ref. 1 we obtain, from (B5) and (B15),

$$
I_{e}+I_{a}=-\int d \overrightarrow{\mathbf{J}} d \overrightarrow{\mathbf{J}}^{\prime} d \overrightarrow{\mathbf{w}}^{\prime} \sum_{K=1}^{M} \omega_{K}\left[\frac{e^{2}}{2 \pi c^{2}}(2 \pi)^{M} \sum_{\overrightarrow{\mathbf{n}}}^{\prime}\left(\overrightarrow{\mathbf{n}} \cdot \vec{\omega}_{0}\right)^{2} \operatorname{Re}\left\{\sigma_{\overrightarrow{0},-\overrightarrow{\mathbf{n}}}\left[J_{K}\right]\left(\overrightarrow{\mathbf{n}} \cdot \vec{\omega}_{0} ; \vec{\omega}_{0}\right)\right\}\left(W_{0}+\pi^{2} \mathscr{Y}^{2} W_{0}^{\prime}\right)\right]
$$

On the other hand, using expressions (2.27) and (3.13), we have

$$
\begin{aligned}
I_{e}+I_{a}= & \int_{0}^{\infty} d \omega\left[I_{e}(\omega)+I_{a}(\omega)\right] \\
=-\int_{0}^{\infty} d \omega \int d \overrightarrow{\mathbf{J}} d \overrightarrow{\mathbf{J}}^{\prime} d \overrightarrow{\mathbf{w}}^{\prime} & \left\{\frac{e^{2}}{2 \pi c^{2}}(2 \pi)^{M} \sum_{K} \omega_{K} \sum_{\overrightarrow{\mathrm{n}}}^{\prime} \delta\left(\omega-\overrightarrow{\mathbf{n}} \cdot \vec{\omega}_{0}\right)\left(\overrightarrow{\mathbf{n}} \cdot \vec{\omega}_{0}\right)^{2} \operatorname{Re}\left[\sigma_{\overrightarrow{0},-\overrightarrow{\mathbf{n}}}\left[J_{K}\right]\left(\overrightarrow{\mathbf{n}} \cdot \vec{\omega}_{0} ; \vec{\omega}_{0}\right)\right]\right. \\
& \left.\times\left(W_{0}+\pi^{2} \mathscr{Y}^{2} W_{0}^{\prime}\right)\right\}
\end{aligned}
$$

where we have used the identity

$$
\sigma[\mathscr{E}]=\sum_{K=1}^{M} \omega_{K} \sigma\left[J_{K}\right]
$$

[see (5.22) of Ref. 1].

From (B17) and (B18) we see that in the stationary state, verifying $W_{0}+\pi^{2} \mathscr{Y}^{2} W_{0}^{\prime}=0$, not only the full expression under the integral is zero, but each term of the sum in $\overrightarrow{\mathbf{n}}$. Therefore, a stronger condition than the radiative balance, which some authors have called "superdetailed balance" 16 or "detail radiative balance,"6 is fulfilled. This superbalance implies also the "detailed balance" which means physically microscopic reversibility. ${ }^{13}$ In order to prove this we must show that each current component of our Fokker-Planck equation-(4.3) of Ref. 1-is zero. In (B17) each term of the sum in $K$ corresponds to the component $J_{K}$ of the current, so that such a component is zero. On the other hand, this result can be generalized to the remaining components of the current because the Fokker-Planck equation may be put in 
the form [see (6.10) and (5.25) of Ref. 1]

$$
0=\sum_{v} \frac{\partial}{\partial h_{v}}\left[\frac{e^{2}}{2 \pi c^{2}}(2 \pi)^{M} \sum_{\overrightarrow{\mathrm{n}}}^{\prime}\left(\overrightarrow{\mathrm{n}} \cdot \vec{\omega}_{0}\right)^{2} \operatorname{Re}\left\{\sigma_{\overrightarrow{0},-\overrightarrow{\mathrm{n}}}\left[h_{v}\right]\left(\overrightarrow{\mathrm{n}} \cdot \vec{\omega}_{0}, \vec{\omega}_{0}\right)\right\}\left(W_{0}+\pi^{2} \mathscr{Y}^{2} W_{0}^{\prime}\right)\right],
$$

so that each component of the current is zero separately.

As may be seen in Eqs. (B17) and (B20), the detailed balance corresponds essentially to the equality of the absorbed and the emitted power for each orbit in such a way that each term of the sum in $K$ in Eq. (B17) vanishes separately. Each $K$ term represents the net variation of the energy due to the constant of motion $J_{K}$. On the other hand, the radiative balance corresponds to the equality of the absorbed and the emitted power for each frequency [see Eq. (B18)].

${ }^{1}$ R. Blanco, L. Pesquera, and E. Santos, Phys. Rev. D $\underline{27}, 1254$ (1983).

${ }^{2}$ See, for instance, O. Theimer, Phys. Rev. D 4, 1597 (1971); T. H. Boyer, Phys. Rev. 182, 1374 (1969).

${ }^{3}$ T. H. Boyer, Phys. Rev. A 18,1228 (1978).

${ }^{4}$ L. Pesquera and P. Claverie, J. Math. Phys. 23, 1315 (1982).

${ }^{5}$ T. H. Boyer, Phys. Rev. D $\underline{19}, 3635$ (1979).

${ }^{6}$ T. M. Marshall and P. Claverie, Physica (Utrecht) 104A, 223 (1980).

${ }^{7}$ T. W. Marshall and P. Claverie, J. Math. Phys. 21, 1819 (1980); T. W. Marshall, in Mathematical Theory of Dynamical Systems and Microphysics CISM Lecture Course 261, edited by A. Blaquière, F. Fer, and A. Marzollo (Springer, Berlin, 1980).

${ }^{8}$ V. Arnold, Methodes Mathematiques de la Mécanique Classique
(MIR, Moscow, 1976).

9J. D. Jackson, Classical Electrodynamics (Wiley, New York, 1975).

${ }^{10}$ R. Kubo, J. Phys. Soc. Jpn. 12, 570 (1957).

${ }^{11}$ L. Pesquera, Santander thesis, 1980; M. Lax, Rev. Mod. Phys. 38, 541 (1966); P. Claverie, in Proceedings of the Einstein Centennial Symposium on Fundamental Physics, edited by $\mathrm{S}$. Moore et al. (Uniandes, Bogotá, 1981).

${ }^{12}$ N. Van Kampen, Phys. Rep. 24C, 171 (1976).

${ }^{13}$ H. Haken, Rev. Mod. Phys. 47, 67 (1975).

${ }^{14}$ L. de La Peña, J. L. Jimenez, and R. Montemayor, Nuovo Cimento 69B, 71 (1982).

${ }^{15}$ N. G. Van Kampen, Physica (Utrecht) 74, 215 (1974); 74, 239 (1974).

${ }^{16}$ L. de la Peña (unpublished). 